\title{
A high-accuracy and high-efficiency digital volume correlation method to characterize in-vivo optic nerve head biomechanics from optical coherence tomography
}

\footnotetext{
${ }^{1}$ Department of Ophthalmology, University of Pittsburgh, Pittsburgh, PA, USA

2 Department of Bioengineering, University of Pittsburgh, Pittsburgh, PA, USA

${ }^{3}$ Department of Preventive and Restorative Dental Sciences, UCSF, San Francisco, CA, USA

${ }^{4}$ Devers Eye Institute, Legacy Health Research, Portland, OR, USA
}

Short Title: DVC for in-vivo optic nerve head biomechanics

* Correspondence:

Ian A. Sigal, Ph.D.

Laboratory of Ocular Biomechanics

Department of Ophthalmology, University of Pittsburgh School of Medicine 203 Lothrop Street, Eye and Ear Institute, Room 930, Pittsburgh, PA 15213

Phone: (412) 864-2220; Fax: (412) 647-5880

Email: ian@OcularBiomechanics.com

www.OcularBiomechanics.com

Key Words: digital volume correlation (DVC); optic nerve head (ONH); optical coherence tomography (OCT); biomechanics characterization; rigid body motion

Conflict of Interest: Junchao Wei contributed to this work while he was at the University of Pittsburgh. He now works at Konica Minolta Laboratory. Other authors have no conflicts of interest.

Funding: Supported in part by National Institutes of Health R01-EY023966, R01-EY025011, R01-EY028662, P30-EY008098 and T32-EY017271 (Bethesda, MD), the Eye and Ear Foundation (Pittsburgh, PA), and Research to prevent blindness. 


\section{Abstract}

In-vivo optic nerve head $(\mathrm{ONH})$ biomechanics characterization is emerging as a promising way to study eye physiology and pathology. We propose a high-accuracy and high-efficiency digital volume correlation (DVC) method for the purpose of characterizing the in-vivo ONH deformation from volumes acquired by optical coherence tomography (OCT). Using a combination of synthetic tests and analysis of OCTs from monkey ONHs subjected to acute and chronically elevated intraocular pressure, we demonstrate that our proposed methodology overcomes several challenges for conventional DVC methods. First, it accounts for large ONH rigid body motion in the OCT volumes which could otherwise lead to analysis failure; second, sub-voxel-accuracy displacement can be guaranteed despite high noise and low image contrast of some OCT volumes; third, computational efficiency is greatly improved, such that the memory consumption of our method is substantially lower than with conventional methods; fourth, we introduce a parameter measuring displacements confidence. Test of image noise effects showed that the proposed DVC method had displacement errors smaller than 0.028 voxels with speckle noise and smaller than 0.037 voxels with Gaussian noise; The absolute (relative) strain errors in the three directions were lower than $0.0018(4 \%)$ with speckle noise and than $0.0045(8 \%)$ with Gaussian noise. Compared with conventional DVC methods, the proposed DVC method had substantially improved overall displacement and strain errors under large body motions (lower by up to $70 \%$ ), with $75 \%$ lower computation times, while saving about $30 \%$ memory. The study thus demonstrates the potential of the proposed technique to investigate $\mathrm{ONH}$ biomechanics.

Keywords: Digital volume correlation (DVC), Optic nerve head (ONH), Optical coherence tomography (OCT), Deformation characterization, Rigid body motion 


\section{Introduction}

60

The biomechanics of the optic nerve head $(\mathrm{ONH})$ in the posterior pole of the eye play a central role in several pathologies, and are therefore important to prevent blindness [1, 2]. In glaucoma, for instance, increases in intraocular pressure (IOP) have been causally associated with higher risk of neural tissue damage and the consequent vision loss [3,4]. The mechanisms by which higher IOP contributes to the neuropathy are not fully understood, but are known to involve IOP-induced deformations of the retinal ganglion cell axons as they pass through the $\mathrm{ONH}$ [5]. Improved diagnosis and treatment of glaucoma, and of other biomechanics-related ocular pathologies, therefore requires an accurate and efficient method to measure $\mathrm{ONH}$ biomechanics in vivo $[6,7]$.

Optical coherence tomography (OCT) has emerged over the last decade as the most widely used tool to image the $\mathrm{ONH}$ in vivo [8-17]. OCT allows acquiring three-dimensional (3D) volumes of the $\mathrm{ONH}$ with $\mu \mathrm{m}$-scale resolution, and with sufficient signal penetration to visualize the lamina cribrosa region within the $\mathrm{ONH}$. The lamina cribrosa is where glaucomatous neural tissue degeneration starts and is therefore of crucial interest in early diagnosis and treatment of this pathology $[5,18,19]$.

Also substantially advanced over the last couple of decades is the image analysis technique of digital volume correlation (DVC) [20]. DVC allows identifying and tracking corresponding points in multiple image volumes. DVC can thus be used to extract the 3D displacements and deformations between two OCT image volumes, an undeformed reference volume, and a deformed volume. The application of DVC to the analysis of OCT images of the ONH has thus emerged as a promising way to quantify the biomechanical effects caused by increases in IOP, changes in gaze, altered intracranial pressure, loss of blood pressure, and other changes in the eye biomechanical environment of potential pathologic significance [7, 21-27]. However, use of DVC for posterior pole biomechanics is hampered by the difficulty in quantifying $\mathrm{ONH}$ deformations from OCT volumes accurately and efficiently using existing DVC techniques originally developed other purposes. Specifically, we highlight four challenges: First, no two OCT scans are of precisely the same region in 
85 the exact same orientation. Thus, OCT volumes acquired at different times often exhibit both deformations and rigid body motion (i.e., translation and rotation) [28]. Efficient DVC requires defining an anticipated largest rigid body motion. If the rigid body motion is larger than this anticipated value, it will likely not be registered accurately. To avoid this problem it is possible to increase the size of the maximum anticipated motion, but this rapidly decreases computational efficiency. Large rotations, in particular, can also have substantial detrimental effects on the correlations, reducing the accuracy of measurements [29]. Although some motion can be reduced using motion tracking techniques, such as those in Heidelberg's Spectralis, the movements are not entirely removed. Second, OCT volumes contain considerable noise compared with other imaging techniques often used for DVC. OCT scans of the $\mathrm{ONH}$ often have low image contrast, compounded by high speckle noise, which worsen with tissue depth further complicating analysis of deeper structures such as the lamina cribrosa, that are of great interest. Collectively, this hinders convergence of the 3D inverse-compositional Gaussian Newton (ICGN) algorithm used in DVC [30], reducing accuracy. Third, conventional DVC methods are highly demanding in time and computing needs, which may make them impractical. The computational burden results primarily from the need to process a large number of points of interest (POI) within the reference volume and to do a large number of interpolations of the sub-volumes in every iteration of each POI. For example, some previous implementations of DVC for OCT have required between two and fifteen hours to track the displacements of 10,000 to 15,000 points $[7,31]$. In addition, the large number of

103 intermediary parameters needed to avoid redundant calculations increases the memory consumption.

104 Fourth, interpreting DVC results is complicated as it requires simultaneously considering various 105 aspects of the process, such as local image quality and correlation strength. In conventional DVC 106 analyses, correlation coefficients are used implicitly to reflect the confidence of DVC tracked 107 corresponding points. However, if the image region in question has poor contrast, a high correlation 108 coefficient does not necessarily equate with high confidence. If points with low reliability are used to 109 guide the DVC search path, it may compromise the robustness of the DVC computations, and the 110 accuracy of the inferences drawn from the results [32]. 
111 In this work we present a DVC method specifically developed for application on OCT images of the

$112 \mathrm{ONH}$ that addresses the four major challenges mentioned above. Our DVC method uses a combination

113 of rigid body motion correction, sub-voxel point selection, a fast and efficient tricubic interpolation, multi-

114 threaded parallel computations and a novel scalar measure of confidence. Herein, we describe in detail

115 the new techniques and their implementation for the analysis of OCT volumes to quantify IOP-induced

$1163 \mathrm{D}$ deformations of the $\mathrm{ONH}$. We show that the method achieves sub-voxel accuracy when registering

117 corresponding points, in code that runs efficiently in time and computing resources. Notwithstanding its

118 origin, the method fundamentals are not limited to this region or to OCT data, and we expect that the

119 techniques introduced herein will prove useful for DVC in studies of other tissues. 


\section{Methods}

122 Bellow the methods are described as per the following general order: We first describe the process 123 of acquiring OCT volumes of the $\mathrm{ONH}$ in vivo at different states. The proposed DVC method is then 124 described, in this order 1) the pre-registration technique, 2) coarse search to obtain the integer125 voxel corresponding point, 3) sub-voxel registration using 3D IC-GN, 4) a fast and memory saving 126 tricubic B-spline interpolation method, 5) confidence definition for each $\mathrm{POI}$ and its usage to guide 127 the searching path and confidence weighted strain calculation, and 6) the multi-thread parallel 128 computation technique to speed up the computation. Finally, we evaluate the DVC method on artificial volumes with various "synthetic" or preset displacements or strains and on two actual

130 volumes captured at two controlled IOPs, and compare our method with the conventional DVC 131 method. We should note that what we refer to as "The conventional DVC method" herein was 132 composed of the conventional sub-voxel registration method [30], conventional tricubic B-spline 133 interpolation method using a look-up table of control points, the conventional correlation coefficient134 guided searching path, and the conventional strain calculation method, but no pre-registration [33].

\subsection{Optic nerve head scan by an optical coherence tomography}

136 A spectral-domain OCT device (SPECTRALIS OCT2, Heidelberg Engineering, GmbH, Heidelberg,

137 Germany) was used to scan the $\mathrm{ONH}$ in-vivo at different states, i.e., at different longitudinal time 138 points or under different IOP conditions. Scanning was done as described elsewhere [34]. Each

139 OCT scan was comprised of a grid pattern of $768 \times 768$ A-lines, each containing 496 axial samples;

140 the total area was $15 \times 15$ degrees and was centered on the $\mathrm{ONH}$. The axial resolution in tissue is

$141 \quad 3.87 \mu \mathrm{m}$ and the lateral spacing between adjacent A-lines is estimated to be $5.0 \mu \mathrm{m}$ in the monkey 142 retina [34]. The instrument uses averaging in real time to reduce speckle noise, and these scans 143 were obtained with a setting of $\mathrm{N}=5$ sweeps averaged per B-scan. During the acquisition process, 144 a clear, rigid gas-permeable contact lens saturated with $0.5 \%$ carboxymethylcellulose solution was 145 placed over the apex of each cornea. Fig. 1 shows one example of the OCT acquisition of a monkey 
$146 \mathrm{ONH}$. Before DVC analysis, we rescaled the OCT volumes to be isotropic, with the size of the

147 rescaled volumes $768 \times 770 \times 393$ voxels [24, 35].

\section{$148 \quad 2.2$ The DVC method}

150 A semi-automatic pre-registration technique combining manual operation and automatic phase 151 correlation [36] was developed to correct the rigid body motion of the $\mathrm{ONH}$ between volumes, 152 especially the rotation angles of the $\mathrm{ONH}$, ensuring successful DVC correlation analysis. As shown

153 in Fig. 2(a), interactive software with a user interface was developed to help 1) monitor the rigid 154 body motion correction process and 2) manually adjust the position and orientation of the deformed 155 volume to achieve registration with the reference volume. Manual operation was required if the $156 \mathrm{ONH}$ had a large rotation between the reference and deformed volumes. This approach can also 157 help avoid converging to a spurious local optimum. To reduce the burden on the operator, the 158 registration was done on two steps. First, the manual operation which had large error tolerance of 159 rotation angles in the $\mathrm{X}, \mathrm{Y}$, and $\mathrm{Z}$ directions of about 2 degrees made the alignment step fast and easy, while already taking advantage of the operator's experience to avoid errors. Thereafter, phase correlation was used to further correct rigid body motion automatically, and achieve an objective, precise alignment that was independent of the operator's skill. Fig. 2(b) shows the workflow of the presented pre-registration technique. Note that the phase correlation was performed in the Fourier domain due to its high computation efficiency [37]. The translation and rotation were rectified separately in the phase correlation: correcting the translation, then the rotation. The rotation angles in the $\mathrm{X}, \mathrm{Y}$, and $\mathrm{Z}$ directions were also corrected independently: for example, when we correct the 167 rotation angle $\theta_{z}$ in the $\mathrm{Z}$ direction, the other two rotation angles $\theta_{x}$ and $\theta_{y}$ in the $\mathrm{X}$ and $\mathrm{Y}$ directions are presumed as 0 . The detailed calculation process in the phase correlation is illustrated in Section 1 of Supplementary Material 1. Subsequently, a nonlinear optimization method, NelderMead method, was further used to optimize $\left(\theta_{x}, \theta_{y}, \theta_{z}\right)$ by minimizing the $[f(x, y, z)-$ 
$\left.171 g\left(x^{*}, y^{*}, z^{*}\right)\right]^{2}$, where $f$ and $g$ denote the reference and the deformed volumes, respectively:

$$
\left[\begin{array}{l}
x \\
y \\
z
\end{array}\right]=\left[\begin{array}{ccc}
\cos \theta_{z} & \sin \theta_{z} & 0 \\
-\sin \theta_{z} & \cos \theta_{z} & 0 \\
0 & 0 & 1
\end{array}\right]\left[\begin{array}{ccc}
\cos \theta_{y} & 0 & \sin \theta_{y} \\
0 & 1 & 0 \\
-\sin \theta_{y} & 0 & \cos \theta_{y}
\end{array}\right]\left[\begin{array}{ccc}
1 & 0 & 0 \\
0 & \cos \theta_{x} & \sin \theta_{x} \\
0 & -\sin \theta_{x} & \cos \theta_{x}
\end{array}\right]\left[\begin{array}{l}
x^{*} \\
y^{*} \\
z^{*}
\end{array}\right]
$$

172 In practice, we can also start the next round of phase correlation to rectify the rigid body motion

173 again until the deformed volume registers well to the reference volume, as shown in Fig. 2(b). This

174 process is done iteratively. In our experience, one or two rounds of phase correlation were enough

175 for most situations.

176

177

178

179

180

181

182

183

184

185

186

187

188

\subsubsection{Coarse search to obtain the integer-voxel corresponding point}

As illustrated in Fig. 3, a reference subvolume with the size $(2 M+1)^{3}$ voxels centered at the POI $P_{0}$ was chosen in the reference volume $f(x, y, z)$; its target subvolume was then searched pointwise in the deformed volume $g\left(x^{\prime}, y^{\prime}, z^{\prime}\right)$. Zero-mean normalized sum of squared difference (ZNSSD) was employed to evaluate the similarity between the reference subvolume and the searched subvolume in the deformed volume since ZNSSD is robust to the intensity linear variation, as:

$$
C_{Z N S S D}(u, v, w)=\sum_{i=1}^{N}\left(\frac{f(x, y, z)-f_{m}}{\sigma_{f}}-\frac{g\left(x^{\prime}, y^{\prime}, z^{\prime}\right)-g_{m}}{\sigma_{g}}\right)^{2}
$$

where $\sigma_{f}=\sqrt{\sum_{i=1}^{N}\left[f(x, y, z)-f_{m}\right]^{2}}$ and $\sigma_{g}=\sqrt{\sum_{i=1}^{N}\left[g\left(x^{\prime}, y^{\prime}, z^{\prime}\right)-g_{m}\right]^{2}} \cdot f_{m}$ and $g_{m}$ are the average voxel intensity of the reference and deformed subvolumes, respectively. $(u, v, w)=$ $\left(x^{\prime}, y^{\prime}, z^{\prime}\right)-(x, y, z) . i$ is the voxel number and $N=(2 M+1)^{3}$. The subvolume having the lowest $C_{Z N S S D}$ searched in the deformed volume was the target subvolume and its center $\left(x_{0}^{\prime}, y_{0}^{\prime}, z_{0}^{\prime}\right)$ was regarded as the integer-voxel corresponding point of the POI. As a result, the initial displacement $\left(u_{0}, v_{0}, w_{0}\right)$ with integer-voxel accuracy was the difference between $\left(x_{0}^{\prime}, y_{0}^{\prime}, z_{0}^{\prime}\right)$ and $\left(x_{0}, y_{0}, z_{0}\right)$.

\subsubsection{Sub-voxel registration}

The corresponding points from coarse search with integer-voxel accuracy were not enough to 
calculate the accurate strain. In this work, the popular 3D IC-GN was used to obtain the sub-voxel corresponding point in the deformed image. In the coarse search process, the shape change between the reference subvolume and the target subvolume was not considered; whereas, in the sub-voxel registration process, the first-order shape function along with the warp function $\mathbf{W}$ was employed to describe the shape change between the subvolumes by mapping a point $\left(x_{i}, y_{i}, z_{i}\right)$ around the subvolume center $\left(x_{0}, y_{0}, z_{0}\right)$ in the reference subvolume to its corresponding point $\left(x_{i}^{\prime}\right.$, $\left.y_{i}^{\prime}, z_{i}^{\prime}\right)$ in the deformed subvolume as:

$$
\left[\begin{array}{c}
x_{i}^{\prime} \\
y_{i}^{\prime} \\
z_{i}^{\prime} \\
1
\end{array}\right]=\mathbf{W}(\xi ; \mathbf{p})+\left[\begin{array}{c}
x_{0} \\
y_{0} \\
z_{0} \\
1
\end{array}\right]=\left[\begin{array}{cccc}
1+u_{x} & u_{y} & u_{z} & u \\
v_{x} & 1+v_{y} & v_{z} & v \\
w_{x} & w_{y} & 1+w_{z} & w \\
0 & 0 & 0 & 1
\end{array}\right]\left[\begin{array}{c}
\Delta x \\
\Delta y \\
\Delta z \\
1
\end{array}\right]+\left[\begin{array}{c}
x_{0} \\
y_{0} \\
z_{0} \\
1
\end{array}\right]
$$

197 where $\xi=[\Delta x, \Delta y, \Delta z, 1]^{T} .(\Delta x, \Delta y, \Delta z)$ are the distance from $\left(x_{i}, y_{i}, z_{i}\right)$ to the center $\left(x_{0}, y_{0}, z_{0}\right)$. $198 \mathbf{p}$ represents the deformation parameters between the reference and the deformed subvolumes: $\mathbf{p}$ $199=\left\{u, v, w, u_{x}, u_{y}, u_{z}, v_{x}, v_{y}, v_{z}, w_{x}, w_{y}, w_{z}\right\}$. The first three parameters are the displacement 200 components in the $\mathrm{X}, \mathrm{Y}$, and $\mathrm{Z}$ directions, respectively; while the other six are the displacement derivatives of the subvolume. Note that these six displacement derivatives in $\mathbf{p}$ are generally not as accurate as the gradients of the three displacement components to represent the deformation of the subvolume, thus, the latter was used to characterize the $\mathrm{ONH}$ deformation in this work. By introducing the deformation parameter $\mathbf{p}$ and the warp function $\mathbf{W}$ to consider the shape change 205 between the reference subvolume and the target subvolume, Eq. (2) can be rewritten as

$$
C_{Z N S S D}(\Delta \mathbf{p})=\sum_{i=1}^{N}\left(\frac{f\left(\mathrm{P}_{0}+\mathbf{W}(\xi ; \Delta \mathbf{p})\right)-f_{m}}{\sigma_{f}}-\frac{g\left(\mathrm{P}_{0}^{\prime}+\mathbf{W}(\xi ; \mathbf{p})\right)-g_{m}}{\sigma_{g}}\right)^{2}
$$

where $\Delta p$ is the incremental deformation parameter: $\Delta p=\left\{\Delta u, \Delta v, \Delta w, \Delta u_{x}, \Delta u_{y}, \Delta u_{z}, \Delta v_{x}\right.$, $\left.207 \Delta v_{y}, \Delta v_{z}, \Delta w_{x}, \Delta w_{y}, \Delta w_{z}\right\} . P_{0}$ and $P_{0}^{\prime}$ are the centers of the reference and the deformed 208 subvolumes. The objective of this sub-voxel registration was to calculate the deformation parameter p by iteratively minimizing $C_{Z N S S D}$. The iteration does not stop until the following convergence 
criterion is met:

$$
\begin{gathered}
\|\Delta \mathbf{p}\|=\left[\Delta u^{2}+\Delta v^{2}+\Delta w^{2}+\left(M \Delta u_{x}\right)^{2}+\left(M \Delta u_{y}\right)^{2}+\left(M \Delta u_{z}\right)^{2}+\left(M \Delta v_{x}\right)^{2}+\left(M \Delta v_{y}\right)^{2}\right. \\
\left.+\left(M \Delta v_{z}\right)^{2}+\left(M \Delta w_{x}\right)^{2}+\left(M \Delta w_{y}\right)^{2}+\left(M \Delta w_{z}\right)^{2}\right]^{1 / 2} \leq T_{\Delta \mathrm{p}}
\end{gathered}
$$

211 where $T_{\Delta p}$ is a threshold. In this work, when the number of iterations exceeded 20, we deemed it 212 a failed convergence. The weak texture, low contrast, and high noise level of the OCT volume of 213 the $\mathrm{ONH}$ reduce the convergence ability of the iterations. In order to ensure the accuracy, even if

214 the iterations fail to converge, it is still required to find the sub-voxel-accuracy corresponding point.

215 Two methods are presented in this work. The intermedia $C_{Z N S S D}, W(\xi ; p)$ and $\|\Delta p\|$ in each iteration are recorded. Method 1 is to deduce the sub-voxel-accuracy corresponding point using the $\mathbf{p}$ having the minimal $\|\Delta p\|$. Method 2 is to do that using the $\mathbf{p}$ having the minimal $C_{Z N S S D}$. The

218 first three parameters of the corresponding $\mathbf{p}$ are the sub-voxel displacement vector. Fig. 4 shows 219 the workflow of the 3D IC-GN iteration method.

221 A large number of non-integer voxel interpolations are required to calculate the wrapped deformed subvolume in the 3D IC-GN iteration process. Due to the low accuracy of the trilinear interpolation method, in this work, the tricubic B-spline interpolation method is used to calculate the voxel intensity at the non-integer voxel position [38]. Nevertheless, the computation intensity of the conventional tricubic B-spline interpolation method is very high and it consumes a lot of memory to save the look-up table. The objective of the proposed interpolation method is to improve the computation efficiency and save the memory. As shown in Fig. 5, the presented tricubic B-spline 228 interpolation can be divided into four bicubic B-spline interpolation and one cubic B-spline 229 interpolation; each bicubic B-spline interpolation can further be divided into five cubic B-spline 230 interpolation. Hence, each tricubic B-spline interpolation is composed of 21 cubic B-spline 231 interpolations. The cubic B-spline interpolation is the basis, in which the interpolated value can be 232 calculated by 


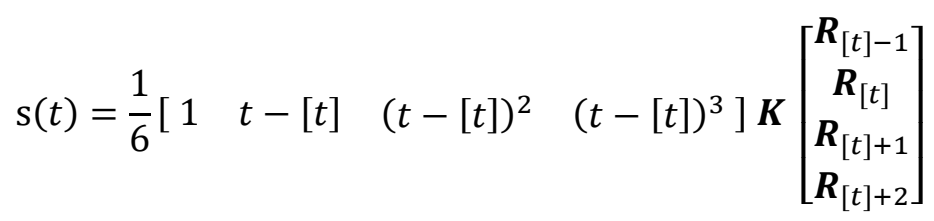

233 where $[\cdot]$ is the integer function. $t$ is the position to be interpolated. $K$ is a $4 \times 4$ interpolation kernel 234 and it is set as $[1,4,1,0 ;-3,0,3,0 ; 3,-6,3,0 ;-1,3,-3,1]$. $R$ denotes the control points determined 235 by the discrete values $\boldsymbol{Q}$ at the regular nodes as follows:

$$
A R=6 Q
$$

236 where $\boldsymbol{A}$ is the coefficient matrix. In this work, we integrate Eq. (7) into Eq. (6) by separating only 237 four elements from $\boldsymbol{Q}$ to calculate the necessary control points $\boldsymbol{R}$ used in Eq. (6), as follows:

$$
\mathrm{s}(t)=\left[\begin{array}{llll}
1 & t-[t] & (t-[t])^{2} & (t-[t])^{3}
\end{array}\right] \boldsymbol{K}\left(\boldsymbol{A}_{\mathbf{4} \times \mathbf{4}}\right)^{-\mathbf{1}}\left[\begin{array}{c}
\boldsymbol{Q}_{[t]-1} \\
\boldsymbol{Q}_{[t]} \\
\boldsymbol{Q}_{[t]+1} \\
\boldsymbol{Q}_{[t]+2}
\end{array}\right]
$$

238 If the boundary condition is set as $\boldsymbol{R}_{[t]-1}=\boldsymbol{Q}_{[t]-1}$ and $\boldsymbol{R}_{[t]+1}=\boldsymbol{Q}_{[t]+1}$ for each interpolation, the $2394 \times 4$ coefficient matrix $\boldsymbol{A}$ becomes $[6,0,0,0 ; 1,4,1,0 ; 0,1,4,1 ; 0,0,0,6]$. In order to speed up 240 the computation, we build up a look-up table for the results of the first three multiply factors: $L U T=$

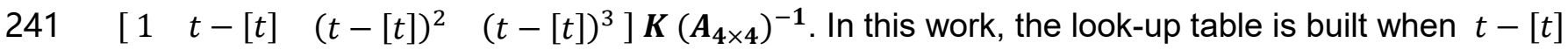
242 increases from 0 to 1 at the step of $\Delta t=0.00005$. Hence, LUT has the size $20001 \times 4$ and only 243 occupies $0.61 \mathrm{MB}$ if the data is saved as double precision type. It is noted that LUT is invariant to 244 the discrete values $\boldsymbol{Q}$, indicating no need to update the look-up table if the processed data is 245 changed. Since one cubic B-spline in our work only includes four multiply calculation and three 246 additive calculation, each non-integer voxel interpolation is involved with 84 multiply calculation and 24763 additive calculation, indicating a relatively low computation intensity of the proposed 248 interpolation method. 
250 In the conventional DVC method, the correlation coefficient $C o r r=1-C_{Z N S S D} / 2$ is directly used to 251 reflect the confidence of the DVC tracked corresponding point and the larger Corr indicates higher

252 confidence. The corresponding points with higher confidence are then used to guide the searching 253 path. The position of the high reliable corresponding point can be utilized to estimate the positions 254 of its surrounding unsearched POls, thereby reducing the searching area greatly and enhancing 255 the searching efficiency. However, in practice, the POI in the low contrast region even with large 256 Corr sometimes has incorrect corresponding point, indicating that the large Corr of the POI in the 257 low contrast region does not mean its high confidence. Using low reliable POls to guide the 258 searching path would negatively affect its neighboring unsearched POls. The average voxel 259 intensity gradient (AVIG) is a good indicator to the image contrast; the larger AVIG generally 260 represents the higher contrast [39]. In this work, we combine both the Corr and AVIG to define the 261 confidence Conf of the POI as follows:

262 If $\operatorname{Corr}_{i}>T_{\text {corr }}$ (a threshold, it set as 0.72) or $\operatorname{Corr}_{i}<T_{\text {corr }} \& A V I G_{i}<$ Const:

$$
\operatorname{Conf}_{i}=\operatorname{Corr}_{i} \times\left(\frac{A V I G_{i}}{\text { Const }}\right)^{2}
$$

263 Otherwise,

$$
\operatorname{Conf}_{i}=\operatorname{Corr}_{i}
$$

264 Where Const is a constant and set as the $\overline{A V I G} \times 0.65$ based on the experience. ( $\overline{A V I G}$ denotes 265 the average $A V I G$ of all the reference subvolumes). $i$ is the subvolume number. It means that even 266 if $\operatorname{Corr}_{i}$ is large, the $\operatorname{Conf}_{i}$ would still be compromised if its $A V I G_{i}$ is smaller than Const. If Corr $_{i}$ 267 is smaller than the threshold $T_{\text {corr }}$, it implies low reliability and $\operatorname{Conf}_{i}$ would not be larger than $268 \operatorname{Corr}_{i}$ even if its $A V I G_{i}$ is large. Subsequently, the POI with the Conf higher than a threshold $269 T_{\text {conf }}$ is then used to guide the searching path. 
271 Although rigid body motion is greatly corrected by the pre-registration technique (subsection 2.2.1),

272 it is possible that it was not completely removed. Hence, the remaining small rigid body motion

273 should be further removed based on the tracked corresponding points before the strain calculation

274 [40]. The derivatives of the displacement vector in the $X, Y$, and $Z$ directions are the stain

275 components. However, the direct derivative calculation is highly sensitive to the noise, resulting in

276 large strain error. Alternatively, in the Savitzky-Golay filter-based method (SGFM), the strains are

277 calculated by fitting a cloud of neighboring discrete displacement vectors within a predefined cuboid

$278\left(2 N_{1}+1\right) \times\left(2 N_{2}+1\right) \times\left(2 N_{3}+1\right)$, namely strain calculation box thereafter, using the least square method

279 (LSM). Since the noise in the local displacement field can be greatly suppressed during the fitting

280 process, the strains calculated in this way are much more accurate than those from the direct

281 derivative calculation. In addition, we used the confidence Conf of the POI to weight every element

282 in the displacement field. The strains $\left(\frac{\partial u}{\partial x}, \frac{\partial u}{\partial y}, \frac{\partial u}{\partial z}, \frac{\partial v}{\partial x}, \frac{\partial v}{\partial y}, \frac{\partial v}{\partial z}, \frac{\partial w}{\partial x}, \frac{\partial w}{\partial y}, \frac{\partial w}{\partial z}\right)$ can be calculated as

283 follows

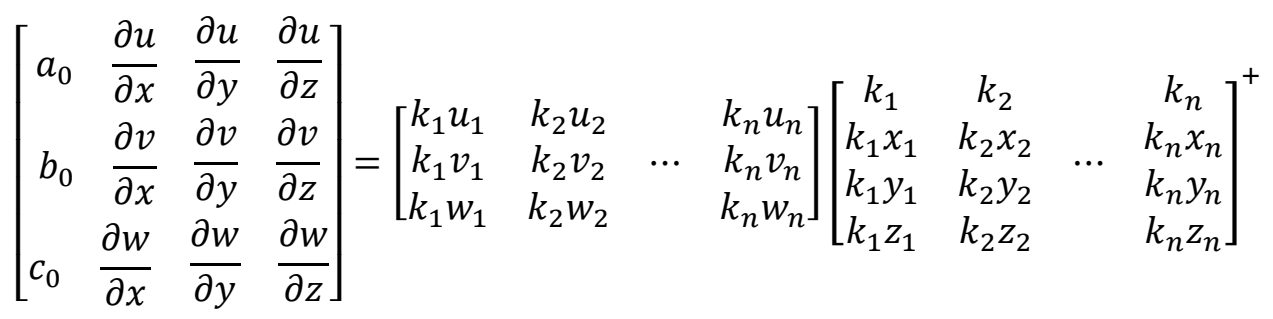

284 where $k_{i}=\operatorname{Conf}_{i} .[\cdot]^{+}$denotes the right inverse matrix. $a_{0}, b_{0}, c_{0}$ are three parameters. $\left(u_{i}, v_{i}\right.$, $\left.285 w_{i}\right)$ and $\left(x_{i}, y_{i}, z_{i}\right)$ are the displacement and position of the $i^{\text {th }} \mathrm{POI}$ in the strain calculation box. 286 The size of the strain calculation box was set as $9 \times 9 \times 9$ in this work.

288 Computation intensity of DVC is very high because the size of the subvolume for correlation is often more than 10000 voxels and a large number of POls have to be searched. Hence, we try to speed up the computation using the multi-thread parallel computation technique. The DVC method was 
291 programmed using C++ in Visual Studio 2019 (Microsoft Corp, Seattle, WA), except for the rigid body motion correction part, which was programmed in python. Eleven computation threads were used in our method. The workflow of the proposed DVC method using multi-thread parallel computation is shown in Section 2 of Supplementary Material 1.

\subsection{Test methods for the proposed DVC technique}

\subsubsection{Artificial rigid body motions to verify the pre-registration technique}

297 An actual OCT volume of a monkey ONH was used as an example (Fig. 6). Three rigid body 298 motions were applied to the OCT volume. Their preset translations $\left[T_{x}, T_{y}, T_{z}\right]$ were the same of [$3.2,1.8,9.3]$ voxels; while the preset rotations $\left[\theta_{x}, \theta_{y}, \theta_{z}\right]$ were $\left[5.3^{\circ},-5.8^{\circ},-14.4^{\circ}\right],\left[-5.3^{\circ}, 5.8^{\circ}\right.$, $\left.14.4^{\circ}\right]$, and $\left[-8.3^{\circ}, 7.6^{\circ},-11.2^{\circ}\right]$, respectively. As noted elsewhere, noise is inevitable in the actual OCT volumes. As speckle noise, a multiplicative noise, is considered the majority noise in the OCT volume [41], we added speckle noise with a standard deviation (SD) of 0.05 into each OCT volume with rigid body motion. Additionally, Gaussian noise, a common noise, was also considered in the test: Gaussian noise with the mean of 0 and the SD of 0.05 was separately added into the OCT volumes. The pre-registration technique was then used to correct the preset rigid body motions. Performance of the pre-registration technique was evaluated by the absolute difference $\left[\Delta T_{x}, \Delta T_{y}\right.$, $\left.\Delta T_{z}, \Delta \theta_{x}, \Delta \theta_{y}, \Delta \theta_{z}\right]$ between the calculated rigid body motion and the preset one. Moreover, we also tested the proposed method on 7 pairs of actual OCT volumes (Supplementary Material 2).

Various rigid sub-voxel translations and strained deformations, including stretch, compression, and shear, were applied to the actual OCT volume, as follows:

312 (1) Rigid sub-voxel translations: $u=v=w=0.2,0.4,0.6$, and 0.8 voxels, respectively.

(2) Stretch: $w_{z}\left(\frac{\partial w}{\partial z}\right)=0.04,0.07$, and 0.10 , respectively; while $u_{x}\left(\frac{\partial u}{\partial x}\right)=v_{y}\left(\frac{\partial v}{\partial y}\right)=-0.016$, - 
315 (3) Compression: $w_{z}=-0.04,-0.07$, and -0.10 , respectively; while $u_{x}=v_{y}=0.016,0.028$, and $316 \quad 0.04$, respectively.

317 (4) Shear deformation: $w_{x}\left(\frac{\partial w}{\partial x}\right)=0.04,0.07$, and 0.10 , respectively; while $u_{z}\left(\frac{\partial u}{\partial z}\right)=v_{z}\left(\frac{\partial v}{\partial z}\right)=-$ $318 \quad 0.016,-0.028$, and -0.04 , respectively.

319 (5) Shear deformation: $w_{x}=-0.04,-0.07$, and -0.10 , respectively; while $u_{z}=v_{z}=0.016,0.028$, 320 and 0.04 , respectively.

321 The Gaussian noise $(S D=0.05)$ and speckle noise $(S D=0.05)$ were also added to each OCT 322 volume, respectively. The proposed DVC method was then used to measure the rigid sub-voxel 323 translation and deformations. The accuracy and computation time of the proposed DVC method 324 were determined.

325 2.3.3 Comparisons between the proposed methods and the conventional methods

326 Several more comparisons between the proposed methods and the conventional methods were 327 done (Supplementary Material 3):

328 (1) the proposed sub-voxel registration methods (Method 1 and Method 2) vs the conventional sub329 voxel registration method;

330 (2) the proposed fast and memory saving tricubic B-spline interpolation method vs the existing 331 methods to build the look-up table of the 64 interpolation coefficients or the control points;

332 (3) the proposed confidence-guided searching method vs the conventional correlation- coefficient333 guided searching method;

334 (4) the proposed confidence weighted strain calculation method vs the conventional strain 335 calculation method.

\subsubsection{Overall performance comparison}

337 Overall performances of the proposed DVC method and conventional DVC method were compared on rigid sub-voxel translations and strained deformation measurement under rigid body motions in accuracy, efficiency, and memory consumption, as follows: 
340 (1) Rigid sub-voxel translations: $u=v=w=0.2,0.4,0.6$, and 0.8 voxels, respectively.

341 (2) Strained deformations: $\left[u_{x}, v_{y}, w_{z}\right]=[-0.04,-0.04,0.1]$ and $[0.04,0.04,-0.1] ;\left[u_{x}, v_{y}, w_{z}\right]=[-$

$3420.04,-0.04,0.1]$ and $[0.04,0.04,-0.1]$

343 In either case, two kinds of rigid body motion were considered: the translations were the same of

$344\left[T_{x}, T_{y}, T_{z}\right]=[2.6,-3.4,4.6]$ voxels; the rotation angles are $\left[\theta_{x}, \theta_{y}, \theta_{z}\right]=\left[2.5^{\circ},-3.3^{\circ}, 3.8^{\circ}\right]$ and $[-$

$3455.1^{\circ},-6.4^{\circ}, 7.3^{\circ}$, respectively. In addition, Gaussian noise $(S D=0.05)$ and speckle noise $(S D=$

$3460.05)$ were also added to each OCT volume, respectively.

\section{2.3.5 Elevated IOP-induced deformation characterization to test the DVC method}

348 Two OCT volumes of a monkey ONH were acquired before and after elevating the IOP from 10 $349 \mathrm{mmHg}$ to $40 \mathrm{mmHg}$ and waiting for $30 \mathrm{~min}$. The reference volume was acquired at $10 \mathrm{mmHg}$, 350 whereas the deformed volume was captured at $40 \mathrm{mmHg}$. The scale ratio of the two volumes: $\mathrm{X}$ 351 pixel size $=4.89 \mu \mathrm{m}$, axial pixel size $=3.87 \mu \mathrm{m}$, distance between scans $=4.90 \mu \mathrm{m}$. The proposed

352 DVC method was then applied to measure the elevated IOP-induced deformation, which was 353 characterized by the maximal and minimal principal strains [31] as well as the maximum shear 354 strain. The respective maximal and minimal strains are referred to as stretch and compression 355 hereafter. The maximum shear strain is half of the absolute difference between the stretch and 356 compression. 


\section{Results}

\subsection{Test results of the presented pre-registration technique}

360 The absolute differences $\left[\Delta T_{x}, \Delta T_{y}, \Delta T_{z}\right]$ between the translations measured by the pre-registration

361 technique and the preset ones were all $[0.2,0.2,0.3]$ voxels, the absolute rotation angle differences

$362\left(\Delta \theta_{x}, \Delta \theta_{y}, \Delta \theta_{z}\right)$ less than $0.4^{\circ}$ (Table 1). Fig.7 shows an example of applying the pre-registration

363 technique to pre-register the volume with a preset rigid body motion to its reference volume. Test

364 of 7 pairs of OCT volumes of actual ONHs are shown in Supplementary Material 2.

\subsection{Test results of artificial rigid sub-voxel translations and strained deformations}

366 Under rigid sub-voxel translations, the displacement errors in the $\mathrm{X}, \mathrm{Y}$, and $\mathrm{Z}$ directions $(\Delta u, \Delta v$,

$367 \Delta w)$ were nearly the same, under 0.037 voxel with Gaussian noise and under 0.028 voxel with

368 speckle noise (Fig. 8(a)). Under strained deformations, average absolute strain errors in the three 369 directions were under 0.0018 with speckle noise and under 0.0048 with Gaussian noise; average 370 relative strain errors under $4 \%$ with Gaussian noise and under $8 \%$ under speckle noise (Fig. 8(b)).

371 Both displacement and strain errors with Gaussian noise were larger than those with speckle noise.

372 In addition, average absolute strain errors tended to increase with the preset deformation; whereas,

373 the average relative strain errors had an opposite tendency. Their computation time was shown in

374 Section 1 of Supplementary Material 3.

\subsection{Overall performance comparison results}

376 Under rigid body motion $\left[T_{x}, T_{y}, T_{z}, \theta_{x}, \theta_{y}, \theta_{z}\right]=\left[2.6,-3.4,4.6,2.5^{\circ},-3.3^{\circ}, 3.8^{\circ}\right](\mathrm{RBM} 1)$, 377 proposed DVC had the overall displacement errors of under 0.05 voxels with speckle noise and 378 under 0.07 voxels with Gaussian noise, and the overall strain errors of under 0.0025 with speckle 379 noise and 0.006 with Gaussian noise; in comparison, conventional DVC method had the great 380 larger displacement and strain errors (Fig. 9(a) and (b)). Under rigid body motion $\left[T_{x}, T_{y}, T_{z}, \theta_{x}\right.$, $\left.381 \theta_{y}, \theta_{z}\right]=\left[2.6,-3.4,4.6,-5.1^{\circ},-6.4^{\circ}, 7^{\circ}\right](\mathrm{RBM} 2)$, conventional DVC method failed to work, 
382 whereas, the proposed method worked well and its displacement and strain errors did not increase

383 (Fig. 9(a) and (b)). The computation time of conventional DVC method increased from 38 minutes

384 under rigid sub-voxel translations to more than 65 minutes under strained deformations, whereas,

385 the computation time of proposed DVC method increased from 8 minutes under rigid sub-voxel

386 translations to less than 18 minutes under strained deformations (Fig. 9(c)). In addition, the

387 proposed DVC method consumed the memory of about $1.9 \mathrm{~GB}$, whereas, the conventional DVC

388 method occupied more memory of about 2.8 GB. The separate comparison results between the

389 proposed methods and conventional methods are demonstrated in Supplementary Material 3.

\subsection{Results of the elevated IOP-induced deformation characterization}

391 The volumes acquired at IOPs of $10 \mathrm{mmHg}$ and $40 \mathrm{mmHg}$ pre-registered well after using the preregistration technique (Fig. 10). The calculated rigid body motion was the translation of $\left[T_{x}, T_{y}, T_{z}\right]$

$393=[1,2,5]$ voxels and the rotation angle of $\left[\theta_{x}, \theta_{y}, \theta_{z}\right]=\left[-0.28^{\circ}, 2.03^{\circ}, 2.24^{\circ}\right]$. Fig. 11 exhibited the 394 stretch, compression and shear strains obtained from the proposed DVC method and the 395 conventional method. The third column shows their differences. The compression tended to be 396 larger than the stretch. On average, the conventional DVC method estimated larger deformations 397 than our proposed DVC method. Please recall that, by convention, compressions are negative 398 deformations. We show them in absolute values to make things easier to compare. 


\section{Discussion}

401 Accurate characterization of ONH deformations between OCT volumes depends upon high-quality

402 DVC. However, due to challenges in image registration, high noise, unclear accuracy, and 403 considerable computational burden, existing DVC methods often fall short. Our goal was to 404 introduce a revised DVC method that addresses the shortcomings of existing DVC methods 405 through a combination of technical improvements. Four main contributions arise from this work: 1) 406 image pre-registration corrected $\mathrm{ONH}$ rigid body motion well; 2) sub-voxel registration was 407 achieved via a modified 3D IC-GN method, 3) the computational burden was dramatically reduced 408 through use of a custom look-up table and occupied memory was saved, and 4) our DVC method had excellent overall performance in displacement and strain calculation and computation time.

410 The synthesis of these contributions resulted in improved DVC measurement quality and workflow 411 efficiency in real-world application. Below we discuss each of these in more detail.

\section{Contribution 1: Image pre-registration corrected ONH rigid body motion well}

413 No two OCT scans are of precisely the same region in the same orientation, even when the 414 subject is anesthetized. Eye-tracking software of some OCT systems can help reduce motion, but 415 often there are still differences in the location or orientation of the scanned region, for example by 416 breath or pulse, sometimes resulting in considerable ONH rigid body motion between the reference 417 and deformed volumes. If substantial rigid body rotations remain, it could lead to failure of the DVC 418 algorithm. Large displacements reduce computational efficiency. Reported DVC methods do not 419 mention efforts to account for the issue of rigid body motion [7, 30, 33, 42].

420 We have shown that our semi-automatic pre-registration technique works well to correct ONH rigid 421 body motion. When the $\mathrm{ONH}$ has a large rotation (more than $4^{\circ}$ ) between the reference and 422 deformed volumes, manual operation was necessary; otherwise, the automatic correction would

423 fail. Manual operation is easy and convenient due to the relatively large rotation angle error 424 tolerance (about $2^{\circ}$ ), the developed user-friendly interactive software and the distinct edge features 425 in the $\mathrm{ONH}$, like the Bruch's membrane. Phase correlation and Nelder-Mead nonlinear optimization 
426 were then used to further automatic calculate the rigid body motion. The deformed volume

427 registered well to the reference volume after pre-registration. The absolute rotation angle

428 differences $\left(\Delta \theta_{x}, \Delta \theta_{y}, \Delta \theta_{z}\right)$ were all less than $0.4^{\circ}$. This technique has the voxel-level translation

429 accuracy, which can explain why all the absolute translation differences $\left(\Delta T_{x}, \Delta T_{y}, \Delta T_{z}\right)$ were 0.3

430 voxels in the tests. ONH rigid body motions in seven actual examples were all corrected well,

431 indicating the effectiveness of the pre-registration technique.

432 Contribution 2: Sub-voxel registration was achieved via a modified 3D IC-GN method

433 After image pre-registration minimized rigid body motion, a coarse search was done first to 434 locate the corresponding point with voxel level accuracy. Bar-Kochba et al. [42] implemented a coarse search process of DVC in the Fourier frequency domain as it can greatly enhance the search efficiency. However, search accuracy decreases considerably if the deformation is larger than $7 \%$.

437 In practice, the $\mathrm{ONH}$ may be subjected to deformations of more than $10 \%$ in response to elevated 438 IOP [7]. Hence, our coarse search process was performed in the spatial domain to ensure accuracy 439 despite its low efficiency. Then, the popular 3D IC-GN iteration method was used to obtain the 440 deformation parameter $p$ including displacement vector of sub-voxel accuracy by minimizing the 441 ZNSSD coefficient. Girard et al. [7] directly applied a genetic optimization algorithm to determine 442 the deformation gradient tensor and displacement vector by minimizing the ZNSSD coefficient. This 443 method can achieve high accuracy, but its computational efficiency is very low, i.e., 15 hours to 444 process 10,000 POls. Compared with the genetic optimization algorithm which was directly applied 445 to determine the deformation gradient tensor and displacement vector by minimizing the ZNSSD 446 coefficient [7], 3D IC-GN has substantially higher computation efficiency, i.e., less than 18 minutes 447 to process 27, 000 POIs. OCT images can have low contrast and considerable noise. Even when 448 the OCT instrument's signal averaging function is used, the acquired volumes in our practical 449 applications still have relatively high noise level. This issue hinders the convergence of 3D IC-GN 450 iteration. When it fails to converge, we selected the $\mathrm{p}$ at the iteration step having the minimal $\|\Delta p\|$ 451 instead of the minimal $C_{Z N S S D}$ in order to ensure sub-voxel accuracy of DVC. $\|\Delta p\|$ was likely to 
452 be at its minimum when $\mathrm{p}$ most approaches the exact value in the iteration process, whereas, the

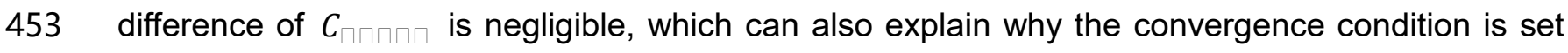

454 based on $\|\Delta p\|$ instead of $C_{\square(p \square \square}$. With the help of the modified 3D IC-GN method for sub-voxel

455 registration, our proposed DVC method had excellent accuracy in sub-voxel translation and

456 strained deformation measurement. The average absolute displacement errors in the $\mathrm{X}, \mathrm{Y}$, and $\mathrm{Z}$

457 directions were very similar, under 0.028 voxels with speckle noise and under 0.037 voxels with

458 Gaussian noise, indicating its isotropic accuracy in the three directions. The respective average,

459 absolute, and relative strain errors in these three directions were less than 0.0018 and $4 \%$ with

460 speckle noise, and less than 0.0045 and $8 \%$ with Gaussian noise. Gaussian noise had a more

461 negative effect on DVC accuracy than the same level of speckle noise. In fact, speckle noise of the

462 actual OCT volume was substantially larger than Gaussian noise. As a result, the former practically

463 results in greater DVC measurement error than the latter.

464 Contribution 3: Computational burden of non-integer voxel interpolation was reduced by a

465 custom look-up table and occupied memory was saved

466 Conventional DVC is computation- and time-intensive partly because of a large number of 467 interpolations which are required with sub-voxel registration to calculate non-integer voxel 468 intensities. In each iteration, the number of interpolations is equal to the size of subvolume, up to 46912,000 non-integer voxel interpolations in this work. In other studies, a trilinear interpolation method 470 was employed [7, 42] due to its high computational efficiency and ease of implementation. Yet, its 471 interpolation error is not negligible. The popular higher-order tricubic B-spline interpolation method 472 was used in this DVC method because of its reduced interpolation error, but its main shortcoming 473 has very low computational efficiency. To speed up computation, we built a look-up table to save 474 the results of the first three multiplicative factors of Eq. (8). This look-up table was independent of 475 the processed OCT volume and only occupied 0.61 MB. Each non-integer voxel interpolation in our 476 method consisted of 84 multiplicative and 63 additive calculations. Optimized computation 477 dramatically increased DVC efficiency by more than $50 \%$ and saved the memory by about $30 \%$, 
478 comparing with the conventional method using a look-up table of B-spline control points (more than

479800 MB memory, 212 multiplicative and 155 additive calculations) [33] and conventional method

480 using a look-up table of 64 interpolation coefficient (more than $50 \mathrm{~GB}, 192$ multiplicative and 63

481 additive calculations) [30]. If higher resolution OCT volumes are used, the proposed interpolation

482 method would have a more direct advantage as its look-up table is independent of the volume and

483 remains the same, whereas, the conventional methods would consume more memory.

484 Contribution 4: Our DVC method had excellent overall performance in displacement and

485 strain calculation and computation time

486 With rigid body motion, the overall performance of our DVC method had fairly considerable

487 advantages over the conventional DVC method in displacement and strain measurement accuracy,

488 and computation efficiency. Our DVC method had overall displacement errors smaller than 0.05

489 voxel with speckle noise and 0.07 voxel with gaussian noise, and the overall strain errors of under

$490 \quad 0.0025$ with speckle noise and 0.006 with Gaussian noise. Overall displacement and strain errors

491 of our DVC method were less than $1 / 3$ of the conventional method. Besides, our DVC method takes

492 less than 18 minutes to process 27000 POIs; the computation efficiency was enhanced by about

$49375 \%$. If the rotation angle is relatively large, i.e., up to 7 degrees, the conventional DVC method

494 would fail to work, whereas, it would not affect the proposed DVC method because of the presented

495 pre-registration technique. The excellent performance of our DVC method was also partly the result

496 of the confidence-guided searching strategy and confidence weighted strain calculation: 1) the

497 number of low reliable POls misused to guide the searching path in our method was decreased to

498 under $1 / 3$ of that of conventional correlation-coefficient-guided searching strategy; and the strain

499 obtained from the confidence weighted strain calculation method was also more accurate than the

500 conventional strain calculation method. The confidence was defined by considering both image

501 contrast of subvolume and correlation coefficient.

502 Synthesis in application

503 In application, our DVC method resulted in high quality measurements of ONH deformation. 
504 After using the described pre-registration technique, $\mathrm{ONH}$ images acquired at the elevated IOP of

$50540 \mathrm{mmHg}$ registered very well to their reference volumes acquired at $10 \mathrm{mmHg}$. The measured

$506 \mathrm{ONH}$ deformations were complex, especially in the regions of central laminar cribrosa and 507 sometimes on the periphery. The maximal stretch, compression and shear strains did not always 508 colocalize. There were relatively large differences (up to 0.06) of the measured stretches, 509 compressions, and shears between the proposed DVC method and the conventional DVC method.

510 The conventional DVC method tended to overestimate the $\mathrm{ONH}$ deformation because strain 511 calculation is very sensitive to noise which often exaggerates the calculated strains.

\section{Recommendations}

513 Despite relatively large amplitude noise, we recommend against the use of OCT image pre514 processing with this DVC method except for an image median filter. In particular, image contrast 515 enhancement and histogram normalization should be avoided. These operations are useful for 516 better visualization of OCT volumes, but they change the real voxel intensity variation information 517 which may negatively affect the DVC accuracy. In our tests, we did not apply any image pre518 processing operations to the OCT volumes in order to better reflect the performance of the DVC 519 method. We did not test for the potential effects of signal compensation techniques [43, 44], 520 although our results suggest that these are not necessary. Additionally, a raster, instead of radial, 521 scan model is recommended for $\mathrm{ONH}$ imaging using the OCT because the former allows sampling 522 with the same resolution over the whole volume, leading to consistent DVC measurement accuracy 523 of the whole volume, whereas, the latter reduces in resolution with the distance from the scan's 524 center.

\section{Remaining challenges and considerations}

526 There are some aspects of the proposed DVC method that deserve further consideration. The 527 shadow of the blood vessels in the OCT volume is a challenging issue affecting the measurement 528 accuracy of the POI near the shadow. Several previous studies have used compensation or similar 529 techniques to reduce the shadows $[43,44]$. It remains unclear how much the compensation may 
530 affect DVC-measured displacements and deformations within the shadows and deep within the

531 tissues. We opted instead for identifying the shadow regions and removing them from analysis to

532 attenuate their influence. Image segmentation along with morphological analysis, such as erosion

533 and dilation, was adopted to separate the shadow from the $\mathrm{ONH}$ in this work. Nevertheless, it is

534 difficult to choose the optimal threshold for segmentation: if the threshold is too large, some

535 important regions may be missed; if it is too small, the shadow region is difficult to remove

536 completely. It may be possible to improve this process by using deep learning methods to separate

537 out the shadows or use the OCT-angiography information from the same scans to predict the

538 location of the most problematic shadows. Setting a relatively large size of the subvolume for

539 correlation is also useful to minimize the influence of shadows. Another consideration is the manual

540 component of our semi-automatic pre-registration technique. Effective manual operation more or

541 less depends on the user's experience to identify and coarsely align the ONH's fairly apparent key

542 features. To achieve fully automatic pre-registration, we may apply 3D scale-invariant feature

543 transform (SIFT) [45] feature points to align the ONH in the future. Lastly, we can perform the DVC

544 technique in the graphics processing unit (GPU) platform to further improve its computational

545 efficiency, although the proposed method has sped up the computation greatly compared with the

546 conventional DVC method.

\section{Summary}

$548 \quad$ We present a high-accuracy and high-efficiency DVC technique to characterize in-vivo ONH

549 deformations from OCT volumes. The method has been successfully applied to characterize the

550 deformation of monkey ONHs subjected to acute and chronic IOP elevation [46, 47]. This technique

551 has the potential to help investigate the pathologic mechanism of glaucoma and eventually, to help

552 clinically diagnose glaucoma in its early stages. Although we demonstrate efficacy of this tool for

553 images of the $\mathrm{ONH}$, this DVC method can also be used to characterize the biomechanics of other

554 biological tissues [48-50]. 


\section{References:}

557

558

559

560

561

562

563

564

565

566

567

568

569

570

571

572

573

574

575

576

577

578

579

580

581

582

583

584

585

586

587

588

589

590

591

592

593

[1] I. C. Campbell, B. Coudrillier, and C. Ross Ethier, Biomechanics of the posterior eye: a critical role in health and disease, J. Biomech. Eng. 136(2) (2014).

[2] Y. Ma et al., IOP-induced regional displacements in the optic nerve head and correlation with peripapillary sclera thickness, Exp. Eye Res. 200 (2020) 108202.

[3] H. A. Quigley, R. M. Hohman, E. M. Addicks, R. W. Massof, and W. R. Green, Morphologic changes in the lamina cribrosa correlated with neural loss in open-angle glaucoma, Am. J. Ophthalmol. 95(5) (1983) 673-691.

[4] C. F. Burgoyne, J. C. Downs, A. J. Bellezza, J.-K. F. Suh, and R. T. Hart, The optic nerve head as a biomechanical structure: a new paradigm for understanding the role of IOP-related stress and strain in the pathophysiology of glaucomatous optic nerve head damage, Prog. Retin. Eye Res. 24(1) (2005) 39-73.

[5] J. C. Downs, M. D. Roberts, and I. A. Sigal, Glaucomatous Cupping Of The Lamina Cribrosa: A Review Of The Evidence For Active Progressive Remodeling As A Mechanism, Exp. Eye Res. 93(2) (2012) 133-140.

[6] D. E. Midgett et al., The pressure-induced deformation response of the human lamina cribrosa: Analysis of regional variations, Acta Biomater. 53 (2017) 123-139.

[7] M. J. A. Girard, N. G. Strouthidis, A. Desjardins, J. M. Mari, and C. R. Ethier, In vivo optic nerve head biomechanics: performance testing of a three-dimensional tracking algorithm, J. R. Soc. Interface 10(87) 201320130459.

[8] I. A. Sigal, B. Wang, N. G. Strouthidis, T. Akagi, and M. J. A. Girard, Recent advances in OCT imaging of the lamina cribrosa, Br. J. Ophthalmol. 98(2) (2014) 34-39.

[9] B. Wang et al., Reproducibility of in-vivo OCT measured three-dimensional human lamina cribrosa microarchitecture, PloS One 9(4) (2014) 95526.

[10] B. J. Wong et al., Relationship of Corneal Hysteresis and Anterior Lamina Cribrosa Displacement in Glaucoma, Am. J. Ophthalmol., 212 (2020) 134-143.

[11] S. H. Lee, E. J. Lee, J. M. Kim, M. J. A. Girard, J. M. Mari, and T.-W. Kim, Lamina Cribrosa Moves Anteriorly After Trabeculectomy in Myopic Eyes, Invest. Ophthalmol. Vis. Sci. 61(6) 202036.

[12] N. Y. Q. Tan et al., Changes in the Anterior Lamina Cribrosa Morphology with Glaucoma Severity, Sci. Rep. 9(1) 20196612.

[13] K. M. Ivers et al., In Vivo Detection of Laminar and Peripapillary Scleral Hypercompliance in Early Monkey Experimental Glaucoma, Investig. Opthalmology Vis. Sci., 57(9) 2016 OCT388.

[14] M. A. Fazio, J. K. Johnstone, B. Smith, L. Wang, and C. A. Girkin, Displacement of the Lamina Cribrosa in Response to Acute Intraocular Pressure Elevation in Normal Individuals of African and European Descent, Invest. Opthalmology Vis. Sci., 57(7) 20163331.

[15] P. Bedggood, F. Tanabe, A. M. McKendrick, A. Turpin, A. J. Anderson, and B. V. Bui, Optic nerve tissue displacement during mild intraocular pressure elevation: its relationship to central 
594

595

596

597

598

599

600

601

602

603

604

605

606

607

608

609

610

611

612

613

614

615

616

617

618

619

620

621

622

623

624

625

626

627

628

629

630

631

632 corneal thickness and corneal hysteresis, Ophthalmic Physiol. Opt. J. Br. Coll. Ophthalmic Opt. Optom., 38(4) 2018 389-399.

[16] Y. W. Kim, D. W. Kim, J. W. Jeoung, D. M. Kim, and K. H. Park, Peripheral lamina cribrosa depth in primary open-angle glaucoma: a swept-source optical coherence tomography study of lamina cribrosa, Eye Lond. Engl., 29(10) 2015 1368-1374.

[17] Z. Wu, G. Xu, R. N. Weinreb, M. Yu, and C. K. S. Leung, Optic Nerve Head Deformation in Glaucoma: A Prospective Analysis of Optic Nerve Head Surface and Lamina Cribrosa Surface Displacement, Ophthalmology, 122(7) 2015 317-1329.

[18] C. A. Girkin et al., Racial Differences in the Association of Anterior Lamina Cribrosa Surface Depth and Glaucoma Severity in the African Descent and Glaucoma Evaluation Study (ADAGES), Invest. Ophthalmol. Vis. Sci., 60(13) (2019) 4496-4502.

[19] Y. Agoumi, G. P. Sharpe, D. M. Hutchison, M. T. Nicolela, P. H. Artes, and B. C. Chauhan, Laminar and prelaminar tissue displacement during intraocular pressure elevation in glaucoma patients and healthy controls, Ophthalmology 118(1) (2011) 52-59.

[20] B. Pan and B. Wang, Some recent advances in digital volume correlation, Opt. Lasers Eng. 135 (2020) 106189.

[21] D. E. Midgett, H. A. Quigley, and T. D. Nguyen, In vivo characterization of the deformation of the human optic nerve head using optical coherence tomography and digital volume correlation, Acta Biomater. 96 (2019) 385-399.

[22] M. A. Fazio, M. E. Clark, L. Bruno, and C. A. Girkin, In vivo optic nerve head mechanical response to intraocular and cerebrospinal fluid pressure: imaging protocol and quantification method, Sci. Rep., 8(1) 201812639.

[23] X. Wang et al., In Vivo 3-Dimensional Strain Mapping Confirms Large Optic Nerve Head Deformations Following Horizontal Eye Movements, Invest. Opthalmology Vis. Sci. 57(13) (2016) 5825.

[24] B. Wang et al., In-vivo effects of intraocular and intracranial pressures on the lamina cribrosa microstructure, PLOS ONE 12(11) (2017) 0188302.

[25] P. A. Sibony, J. Wei, and I. A. Sigal, Gaze-Evoked Deformations in Optic Nerve Head Drusen, Ophthalmology, 125(6) (2018) 929-937.

[26] I. A. Sigal, J. L. Grimm, N.-J. Jan, K. Reid, D. S. Minckler, and D. J. Brown, Eye-Specific IOPInduced Displacements and Deformations of Human Lamina Cribrosa, Invest. Opthalmology Vis. Sci., 55(1) (2014) 1.

[27] A. Korneva, E. C. Kimball, J. L. Jefferys, H. A. Quigley, and T. D. Nguyen, Biomechanics of the optic nerve head and peripapillary sclera in a mouse model of glaucoma, J. R. Soc. Interface, 17(173) (2020).

[28] M. A. Fazio et al., Histologic validation of optical coherence tomography-based threedimensional morphometric measurements of the human optic nerve head: Methodology and preliminary results, Exp. Eye Res., 205 (2021) 108475.

[29] F. Zhong and C. Quan, Digital image correlation in polar coordinate robust to a large rotation, 
Opt. Lasers Eng., 98 (2017) 153-158.

634

635

636

637

638

639

640

641

642

643

644

645

646

647

648

649

650

651

652

653

654

655

656

657

658

659

660

661

662

663

664

665

666

667

668

669

670

671

[30] T. Wang, Z. Jiang, Q. Kemao, F. Lin, and S. H. Soon, GPU Accelerated Digital Volume Correlation, Exp. Mech. 56(2) (2016) 297-309.

[31] I. A. Sigal et al., Measuring in-vivo and in-situ ex-vivo the 3D deformation of the lamina cribrosa microstructure under elevated intraocular pressure, in Optical Elastography and Tissue Biomechanics V, San Francisco, United States, Feb. 2018, p. 36. doi: 10.1117/12.2291243.

[32] B. Pan, B. Wang, D. Wu, G. Lubineau, An efficient and accurate 3D displacements tracking strategy for digital volume correlation, Opt. Laser Eng. 58 (2014) 126-135.

[33] B. Pan, D. Wu, and Z. Wang, Internal displacement and strain measurement using digital volume correlation: a least-squares framework, Meas. Sci. Technol., 23(4) (2012) 045002.

[34] B. Fortune, C. F. Burgoyne, G. Cull, J. Reynaud, and L. Wang, Onset and Progression of Peripapillary Retinal Nerve Fiber Layer (RNFL) Retardance Changes Occur Earlier Than RNFL Thickness Changes in Experimental Glaucoma, Investig. Opthalmology Vis. Sci., 54(8) 20135653.

[35] I. A. Sigal, J. S. Schuman, H. Ishikawa, L. Kagemann, and G. Wollstein, A Problem of Proportions in OCT-Based Morphometry and a Proposed Solution, Invest. Ophthalmol. Vis. Sci., 57(2) 2016 484-485.

[36] "Phase correlation," Wikipedia. Nov. 16, 2020. Accessed: Feb. 15, 2021. [Online]. Available: https://en. wikipedia.org/w/index.php?title=Phase_correlation\&oldid=989017909

[37] B. S. Reddy and B. N. Chatterji, An FFT-based technique for translation, rotation, and scaleinvariant image registration, IEEE Trans. Image Process. 5(8) (2016) 1266-1271.

[38] L. Luu, Z. Wang, M. Vo, T. Hoang, J. Ma, Accuracy enhancement of digital image correlation with B-spline interpolation, Opt. Lett. 36(16) (2010) 3070-3072.

[39] B. Pan, Z. Lu, and H. Xie, Mean intensity gradient: An effective global parameter for quality assessment of the speckle patterns used in digital image correlation, Opt. Lasers Eng., 48(4) (2010) 469-477.

[40] P. J. Sousa, J. M. R. S. Tavares, P. J. S. Tavares, and P. M. G. P. Moreira, Correction of rigid body motion in deformation measurement of rotating objects, Measurement, 129 (2018) 436444.

[41] J. M. Schmitt, S. H. Xiang, and K. M. Yung, Speckle in optical coherence tomography: an overview, in Saratov Fall Meeting '98: Light Scattering Technologies for Mechanics, Biomedicine, and Material Science, Mar. 1999, vol. 3726, pp. 450-461.

[42] E. Bar-Kochba, J. Toyjanova, E. Andrews, K.-S. Kim, and C. Franck, A Fast Iterative Digital Volume Correlation Algorithm for Large Deformations, Exp. Mech., 55(1) (2015) 261-274.

[43] M. J. A. Girard et al., Lamina cribrosa visibility using optical coherence tomography: comparison of devices and effects of image enhancement techniques, Invest. Ophthalmol. Vis. Sci., 56(2) (2015) 865-874.

[44] M. J. A. Girard, N. G. Strouthidis, C. R. Ethier, and J. M. Mari, Shadow removal and contrast enhancement in optical coherence tomography images of the human optic nerve head, Invest. 
Ophthalmol. Vis. Sci., 52(10) (2011) 7738-7748.

[45] M. Niemeijer, M. K. Garvin, K. Lee, B. van Ginneken, M. D. Abràmoff, and M. Sonka, Registration of 3D spectral OCT volumes using 3D SIFT feature point matching, in Medical Imaging 2009: Image Processing, 2009, vol. 7259, p. 725911.

676

[46] F. Zhong et al., In-vivo evidence of increased lamina cribrosa compliance at onset of experimental glaucoma in nonhuman primates, Invest. Ophthalmol. Vis. Sci., 62(8) (2021) 1827-1827.

[47] I. A. Sigal et al., In-vivo evidence of lamina cribrosa deformations induced by chronically elevated IOP in non-human primates, Invest. Ophthalmol. Vis. Sci., 62(8) (2021) 1862-1862.

[48] D. E. Midgett, J. L. Jefferys, H. A. Quigley, and Thao. D. Nguyen, The inflation response of the human lamina cribrosa and sclera: Analysis of deformation and interaction, Acta Biomater., 106 (2020) 225-241.

684

[49] V. A. Acosta Santamaría, M. F. García, J. Molimard, and S. Avril, Characterization of chemoelastic effects in arteries using digital volume correlation and optical coherence tomography, Acta Biomater., 102 (2020) 127-137.

[50] C. M. Disney et al., Synchrotron tomography of intervertebral disc deformation quantified by digital volume correlation reveals microstructural influence on strain patterns, Acta Biomater. 92 (2019) 290-304.

690 
bioRxiv preprint doi: https://doi.org/10.1101/2021.08.07.455176; this version posted August 8, 2021. The copyright holder for this preprint (which was not certified by peer review) is the author/funder, who has granted bioRxiv a license to display the preprint in perpetuity. It is made available under aCC-BY-NC-ND 4.0 International license.

692 


\section{Figures and tables}
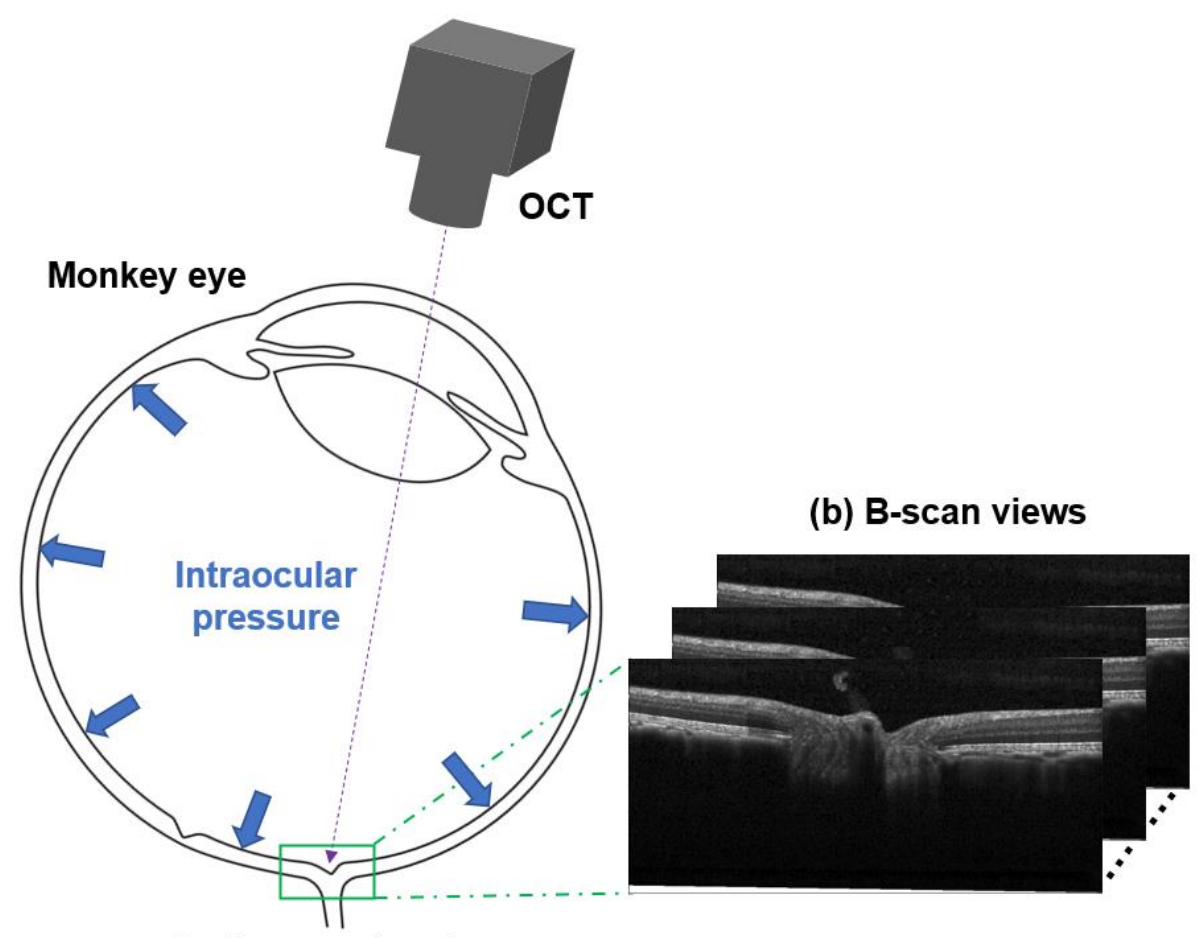

(c) A C-mode view

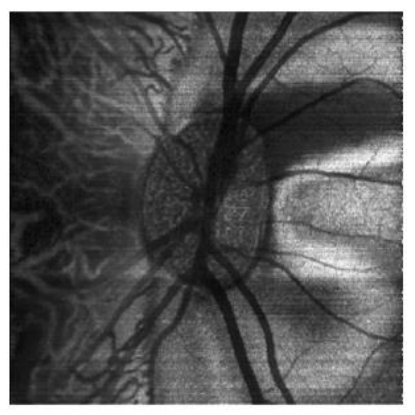

695 Fig. 1. (a) Schematic longitudinal section through a monkey eye. The pressure within the globe is 696 the intraocular pressure (IOP). Optical coherence tomography (OCT) was used to image the optic 697 nerve head $(\mathrm{ONH})$ region in the back of the eye. (b) A series of B-scans acquired from the OCT. (c) 698 A c-mode view of the OCT volume reconstructed by these B-scans. 
(a) User interface
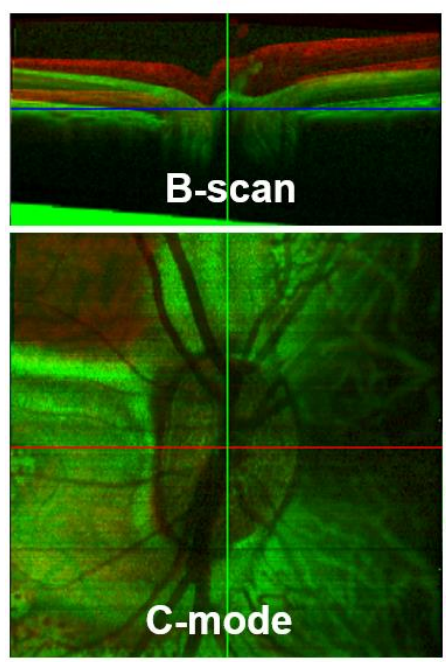

Reference volume

Deformed volume

(1)

(b) Pre-registration workflow

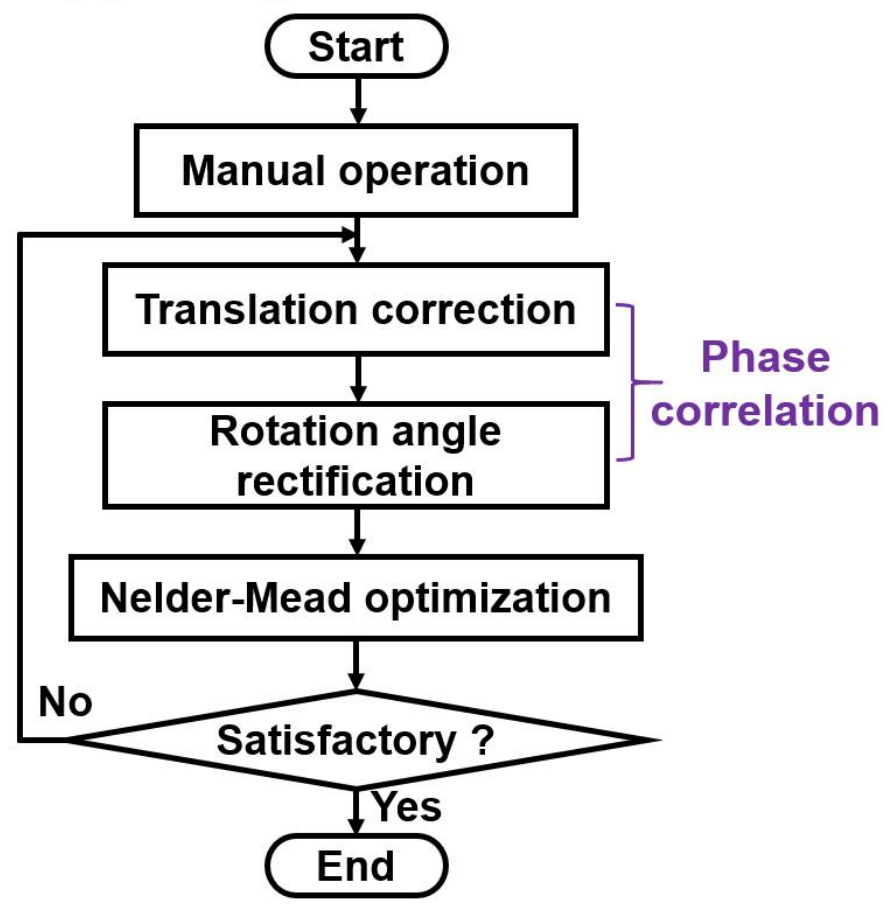

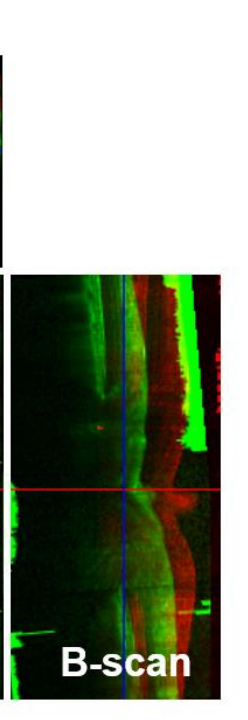


(a) Reference volume $f(x, y, z)$

713 Fig. 3. Schematic illustration of the principle of DVC which is to find the corresponding points

714 between the reference and deformed volume by evaluating the similarity among the subvolumes.

715 The difference between the center $P_{0}$ of the reference subvolume and that center $P_{0}^{\prime}$ of the target

716 deformed volume is the displacement $[u, v, w]^{T} . P_{i}$ and $P_{i}^{\prime}$ are the corresponding points in the

717 reference and deformed subvolumes, respectively. 


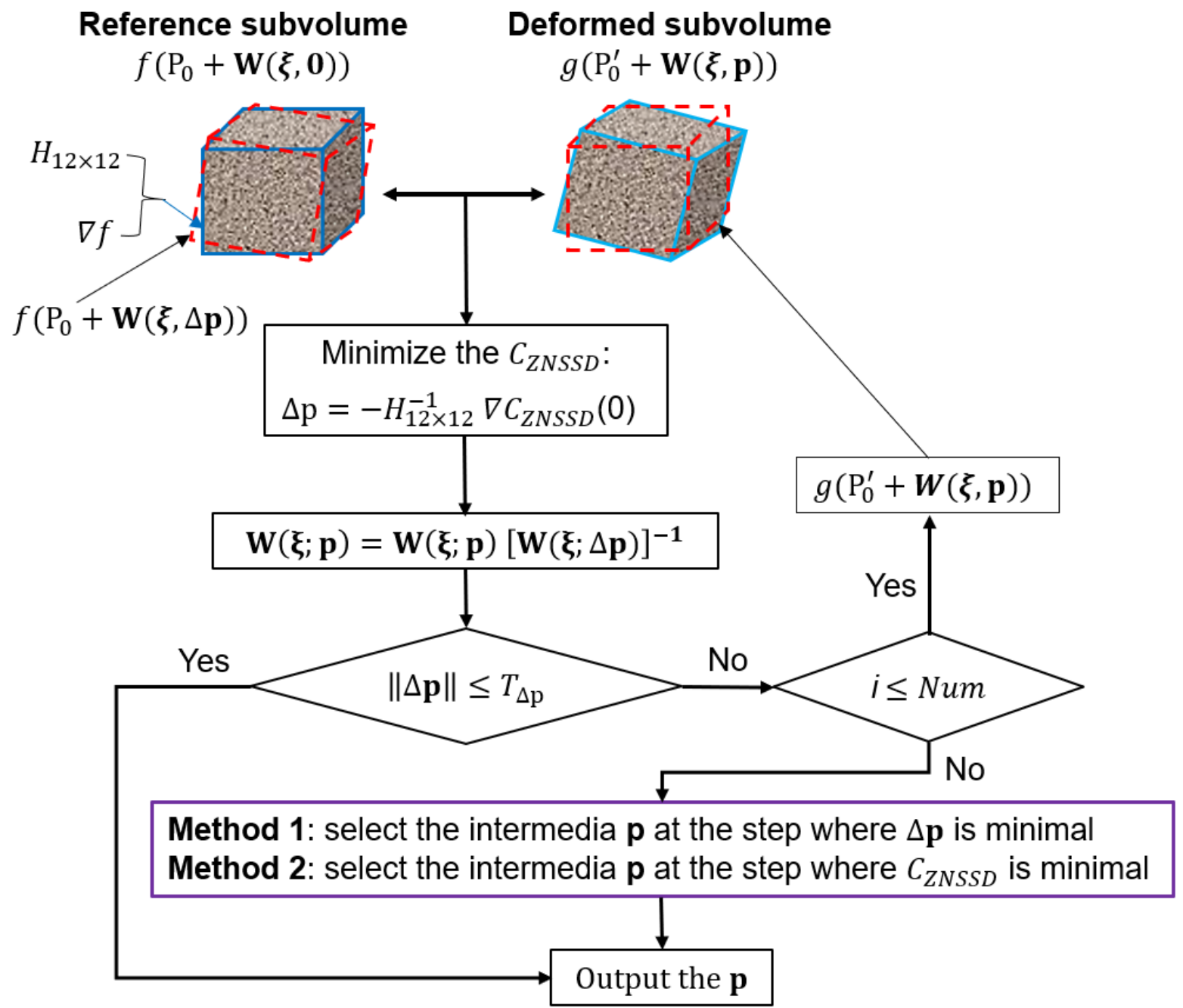

720 Fig. 4. The workflow of the 3D IC-GN iteration method. Due to the weak texture and low contrast, 721 but the large noise of the OCT volume of the ONH, the popular 3D IC-GN iteration method often 722 fails to converge. In the conventional method, when the iteration number exceeds the limit, the corresponding point only has integer-voxel-level accuracy. We present two methods (Method 1 and Method 2) to ensure the sub-voxel accuracy when the iteration number exceeds the limit. 


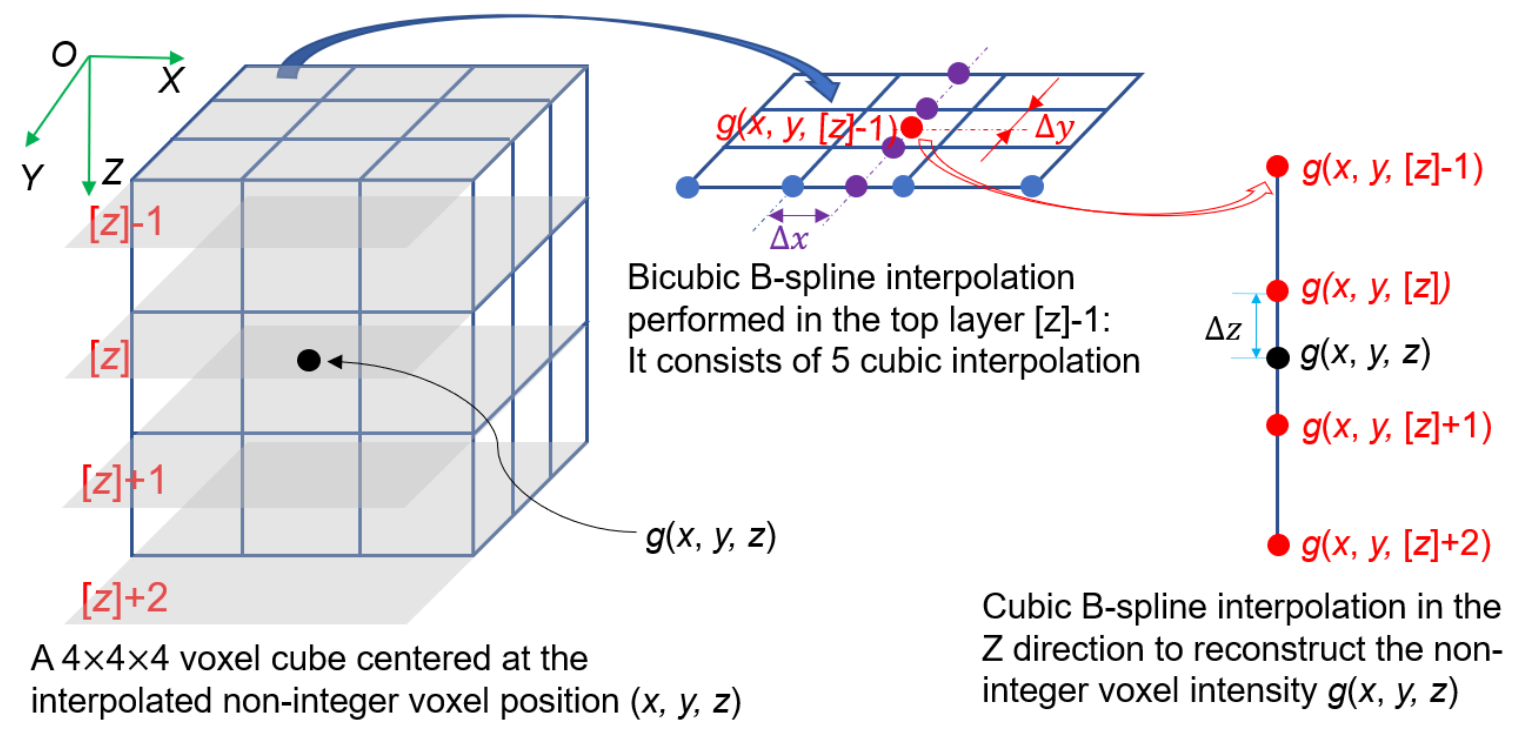
interpolated non-integer voxel position $(x, y, z)$ integer voxel intensity $g(x, y, z)$

727 Fig. 5. Tricubic B-spline interpolation for non-integer voxel intensity calculation. In the proposed 728 method, it is divided into four bicubic interpolations and one more cubic interpolation. Each bicubic 729 interpolation can also be divided into 5 cubic interpolations. Hence, one tricubic B-spline 730 interpolation consists of 21 cubic interpolations. 


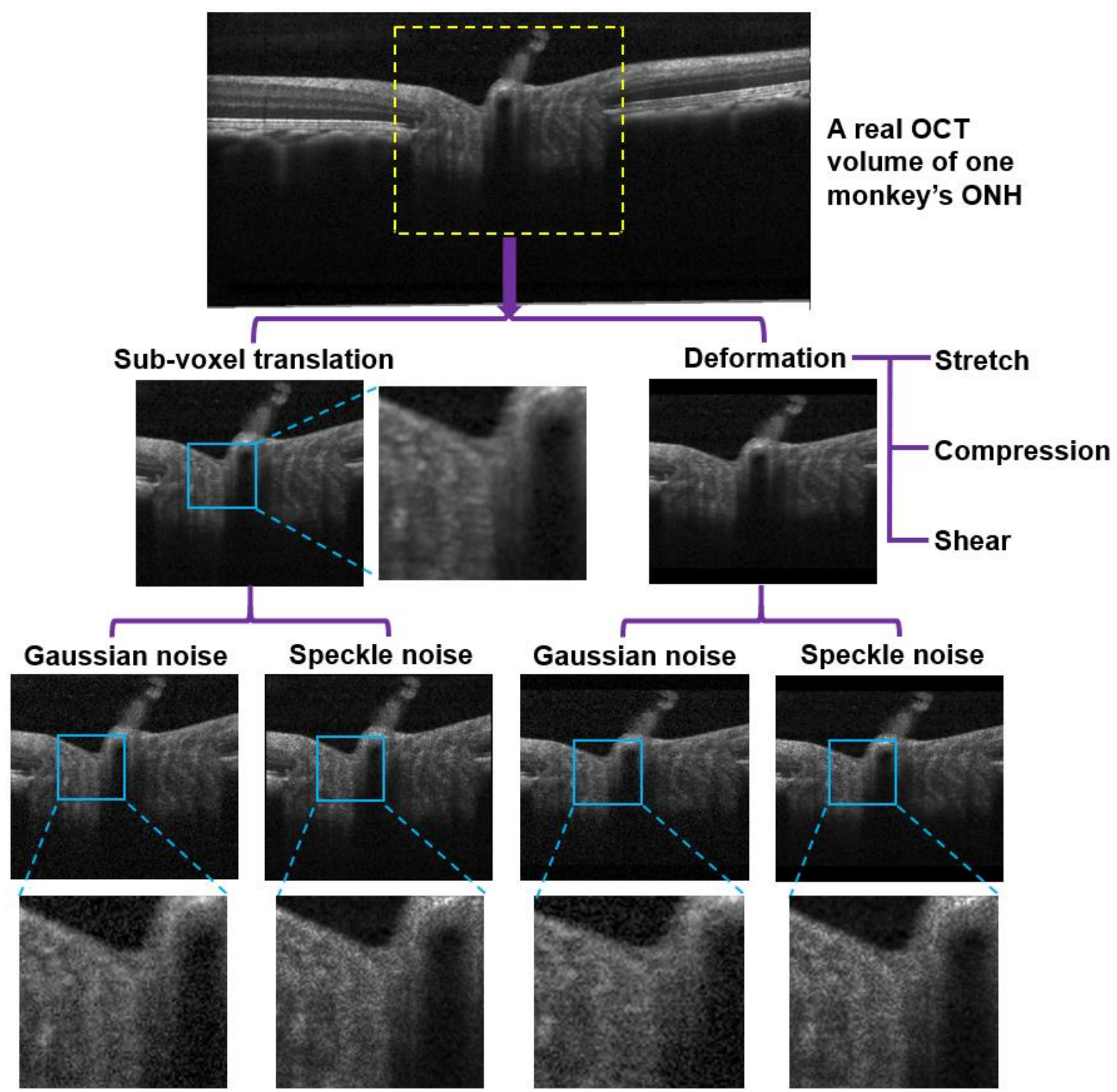

733 Fig. 6. Applying rigid sub-voxel translations and various strained deformations to an actual OCT 734 volume of a monkey's $\mathrm{ONH}$. The region enclosed by the dashed yellow frame was defined the 735 region of interest $(\mathrm{ROI})$ for this test. In addition, Gaussian noise $(\mathrm{SD}=0.05$ ) and/or speckle noise $736(S D=0.05)$ are added into deformed OCT volumes. The close-ups are intended to make it easier

737 to distinguish the noise. The rigid sub-voxel translations $[u, v, w]$ in the $\mathrm{X}, \mathrm{Y}$, and $\mathrm{Z}$ directions are 738 all $0.2,0.4,0.6$, and 0.8 voxel, respectively. The strained deformations are classified into stretch, 739 compression, and shear deformation. 
(a) Before pre-registration
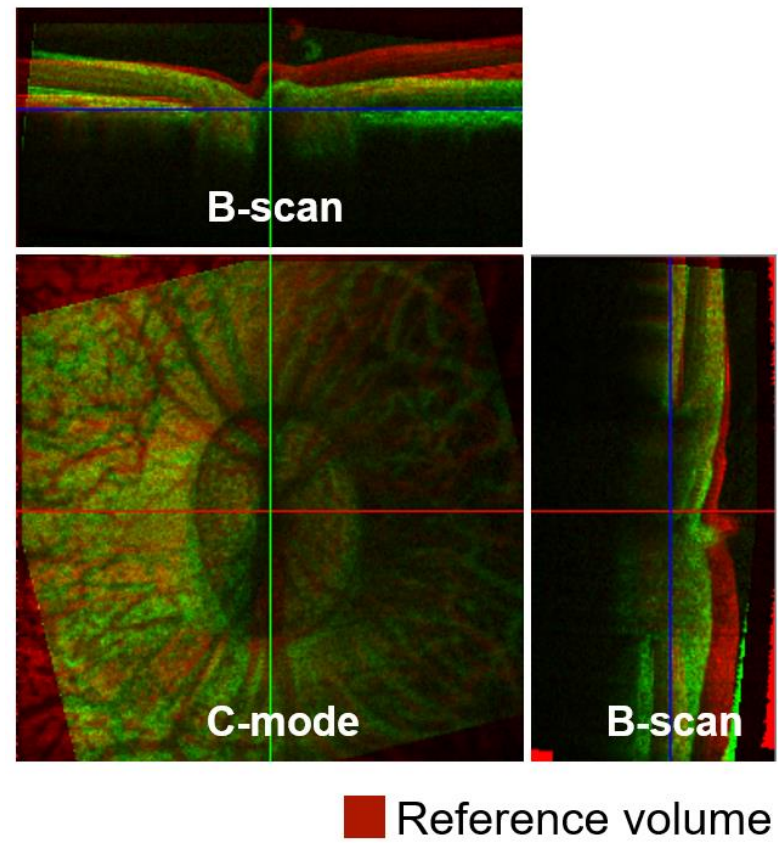

(b) After pre-registration
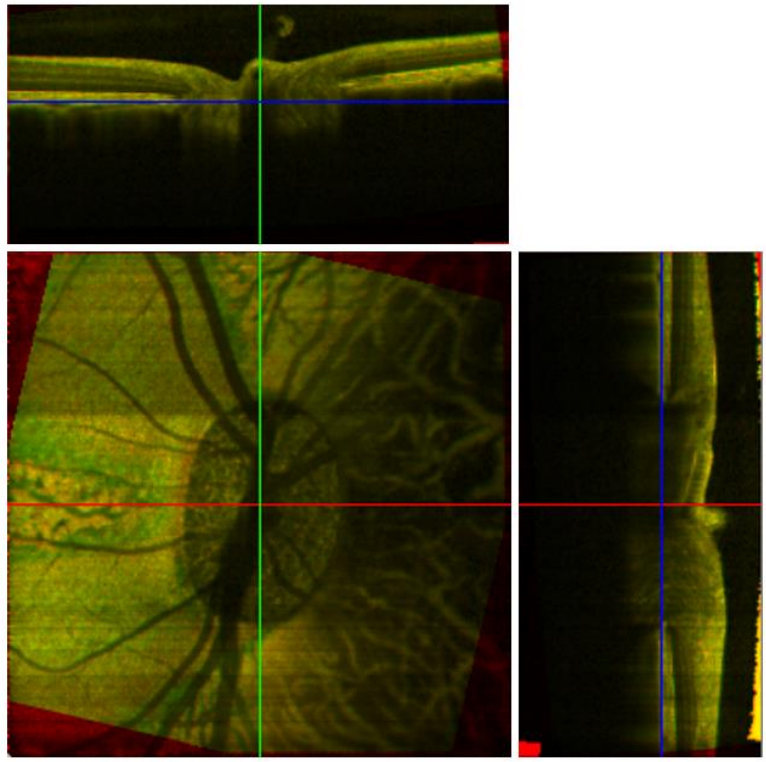

Deformed volume

742 Fig. 7. Test the pre-registration technique on rigid body motion correction. (a) The mapping before 743 pre-registration: the red is the original OCT volume, while the green is the volume with the preset 744 rigid body motion: $\left[T_{x}, T_{y}, T_{z}, \theta_{x}, \theta_{y}, \theta_{z}\right]=\left[-3.2,1.8,9.3,5.3^{\circ},-5.8^{\circ},-14.4^{\circ}\right]$. The added speckle 745 noise level is $S D=0.05$. (b) The mapping after using the pre-registration technique to remove the 746 rigid body motion. When the green voxel registers well its corresponding red voxel, it appears yellow. 
(a) Rigid sub-voxel translation

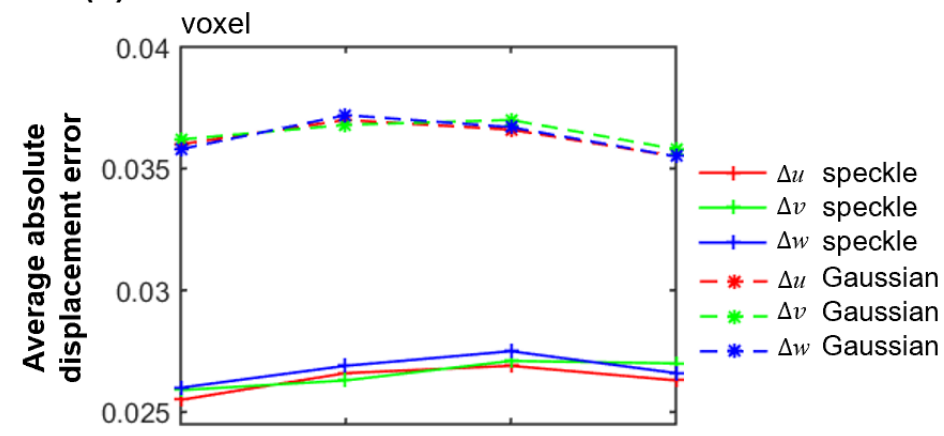

(b) Stretch and compression
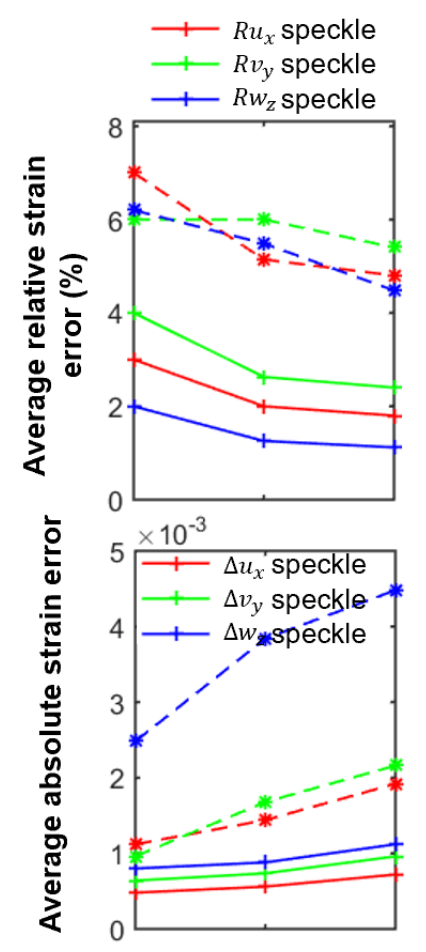

748

749

750

751

752

753

754

$\left[\begin{array}{l}\boldsymbol{u}_{x} \\ \boldsymbol{v}_{\boldsymbol{y}} \\ \boldsymbol{w}_{z}\end{array}\right]=\left[\begin{array}{c}-0.016 \\ -0.016 \\ 0.04\end{array}\right]\left[\begin{array}{c}-0.028 \\ -0.028 \\ 0.07\end{array}\right]\left[\begin{array}{c}-0.04 \\ -0.04 \\ 0.1\end{array}\right]\left[\begin{array}{c}0.016 \\ 0.016 \\ -0.04\end{array}\right]\left[\begin{array}{c}0.028 \\ 0.028 \\ -0.07\end{array}\right] \quad\left[\begin{array}{c}0.04 \\ 0.04 \\ -0.1\end{array}\right]$
- * - Ru $u_{x}$ Gaussian

$-*-R v_{y}$ Gaussian

- * - Rw $w_{z}$ Gaussian

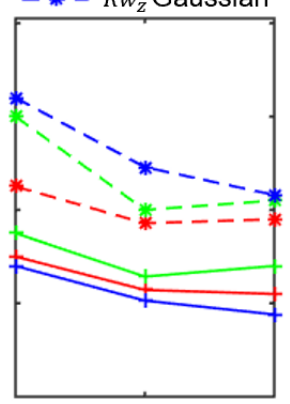

$\times 10^{-3}$

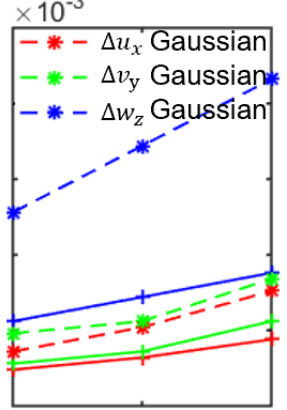

$\left[\begin{array}{l}0.6 \\ 0.6 \\ 0.6\end{array}\right] \quad\left[\begin{array}{l}0.8 \\ 0.8 \\ 0.8\end{array}\right]$

Shear deformation

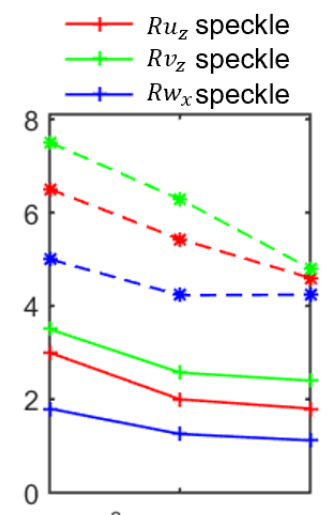

- * - Ru Gaussian

- * - Rv Gaussian

- * $-R w_{x}$ Gaussian
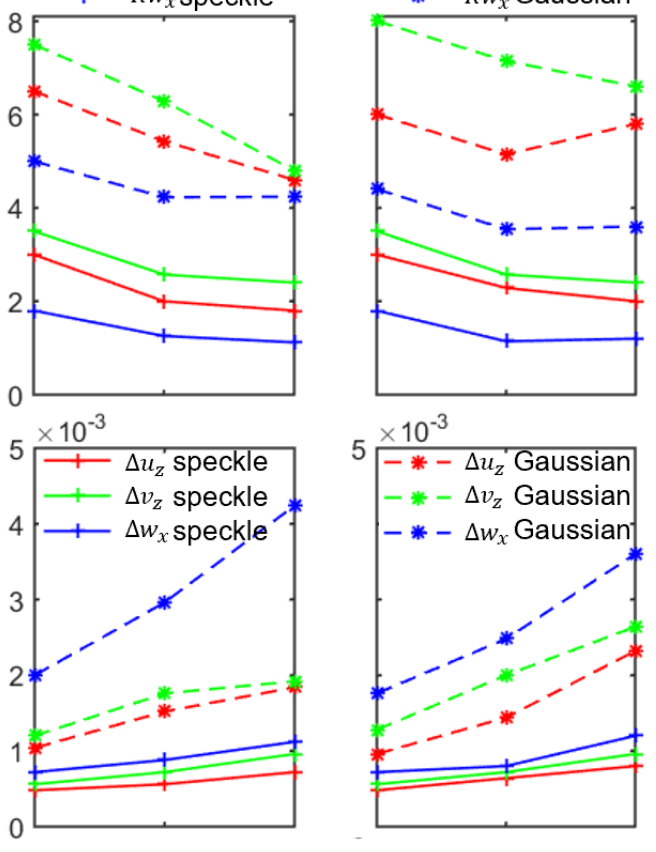

$5 \stackrel{\times 10^{-3}}{--*-\Delta u_{z}^{\prime} \text { Gaussian }}$

- * - $\Delta v_{z}$ Gaussian

$-*-\Delta w_{x}$ Gaussian

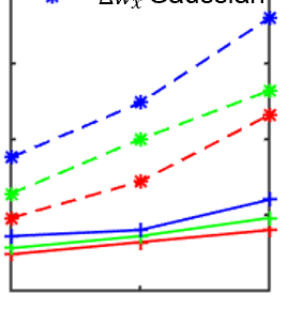

$\left.\begin{array}{l}\boldsymbol{u}_{z} \\ \boldsymbol{v}_{z} \\ \boldsymbol{w}_{x}\end{array}\right]=\left[\begin{array}{c}-0.016 \\ -0.016 \\ 0.04\end{array}\right] \quad\left[\begin{array}{c}-0.028 \\ -0.028 \\ 0.07\end{array}\right]\left[\begin{array}{c}-0.04 \\ -0.04 \\ 0.1\end{array}\right]\left[\begin{array}{c}0.016 \\ 0.016 \\ -0.04\end{array}\right] \quad\left[\begin{array}{c}0.028 \\ 0.028 \\ -0.07\end{array}\right] \quad\left[\begin{array}{c}0.04 \\ 0.04 \\ -0.1\end{array}\right]$

Fig. 8. The test results of the proposed DVC method on rigid sub-voxel translations and various strained deformations in scans with Gaussian or speckle noise. (a) The average absolute displacement errors in the $\mathrm{X}, \mathrm{Y}$, and $\mathrm{Z}$ directions. The preset rigid sub-voxel displacements $(u, v$,

w) are $0.2,0.4,0.6$, and 0.8 voxels, respectively. $(\Delta u, \Delta v, \Delta w)$ denote the measured displacement errors in the $\mathrm{X}, \mathrm{Y}$, and $\mathrm{Z}$ directions, respectively. (b) The average absolute strain errors and the relative strain errors to the preset strains. $\Delta u_{x}$ and $R u_{x}$ denote the respective absolute and 
755 relative strain errors of $u_{x}=\frac{\partial u}{\partial x}$. Other notations were defined in the same way. It can be observed

756 that displacement errors in the $\mathrm{X}, \mathrm{Y}$, and $\mathrm{Z}$ directions are the same. Gaussian noise led to more 757 displacement and strain errors than the same level of speckle noise. The average absolute strain 758 errors increase with the preset strains, whereas, the relative strain errors exhibit the opposite trends. 

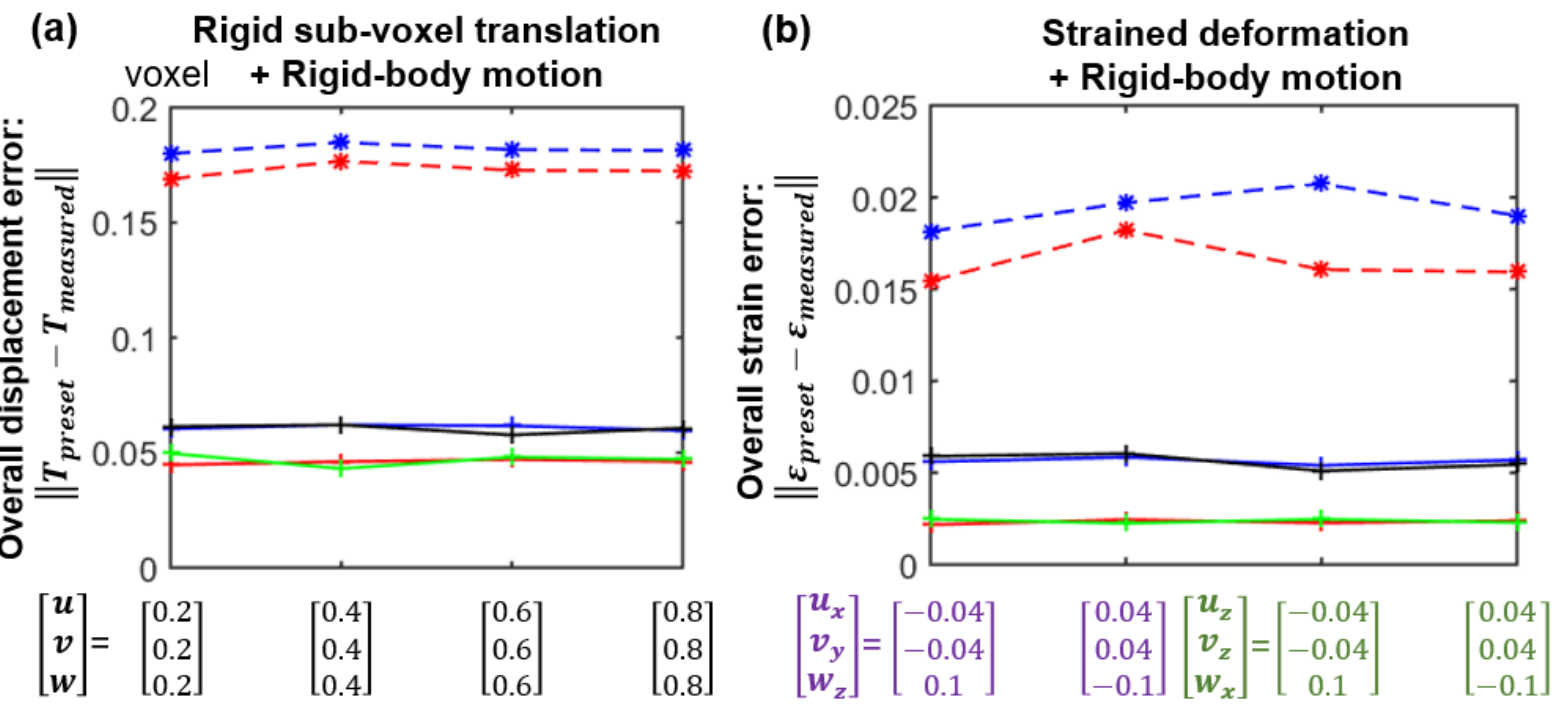

(c)

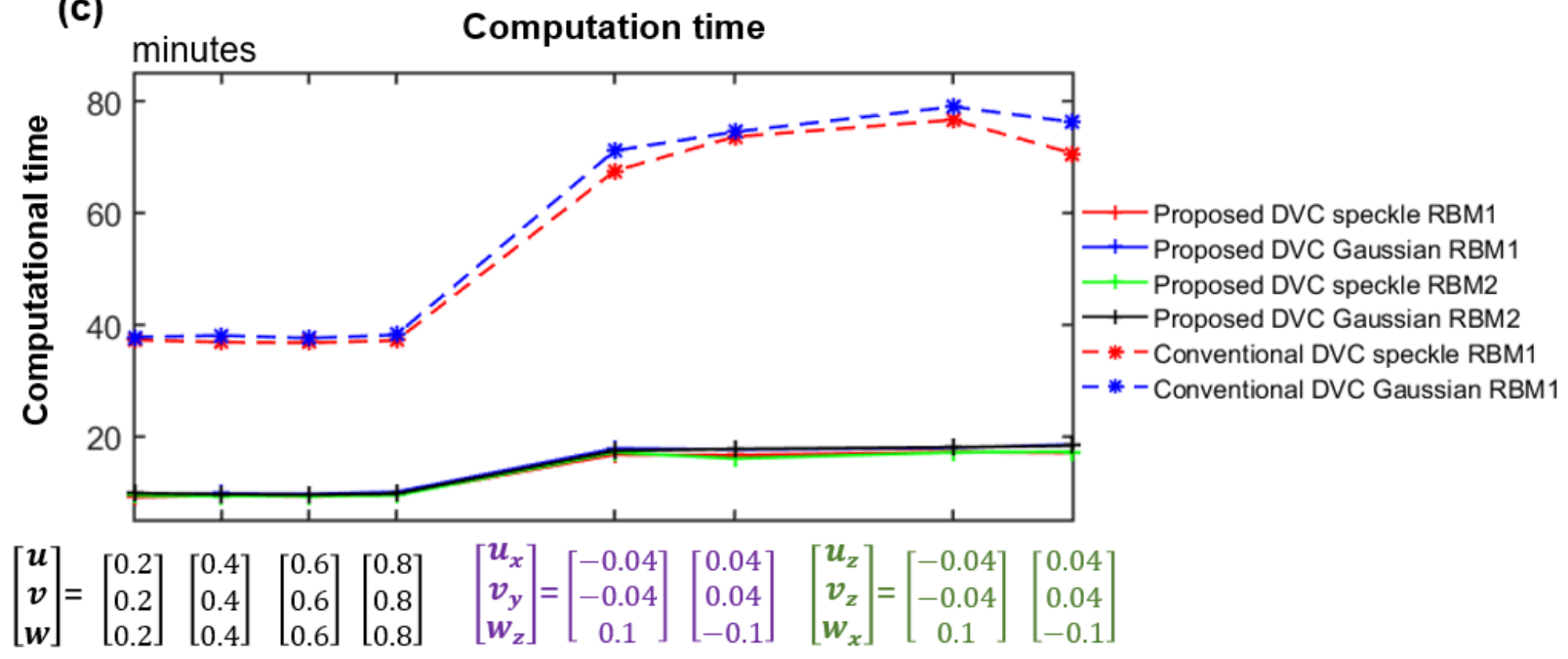

760 Fig. 9. Overall performance comparison between the proposed DVC method and the conventional

761 DVC method on displacement and strain measurement accuracy and computation time under rigid 762 body motion. Two kinds of rigid body motion (RBM1 and RBM2) are set. RBM1: $\left[T_{x}, T_{y}, T_{z}, \theta_{x}\right.$, $\left.763 \theta_{y}, \theta_{z}\right]=\left[2.6,-3.4,4.6,2.5^{\circ},-3.3^{\circ}, 3.8^{\circ}\right]$; RBM2: $\left[T_{x}, T_{y}, T_{z}, \theta_{x}, \theta_{y}, \theta_{z}\right]=\left[2.6,-3.4,4.6,-5.1^{\circ},-\right.$ $7646.4^{\circ}, 7.3^{\circ}$. (a) The overall displacement errors, $T_{\text {preset }}$ can be deduced from the preset rigid sub765 voxel translation and rigid body motion. (b) The overall strain errors. Note that the conventional 766 DVC method failed to work in RMB2, and thus its results are not shown. The results of the proposed 767 DVC method under RBM1 and RBM2 were very similar. It can be seen that, in case of rigid body 768 motion, the proposed DVC method is clearly superior to conventional DVC method in overall displacement error, overall strain error, and computation time. 
(a) Before pre-registration
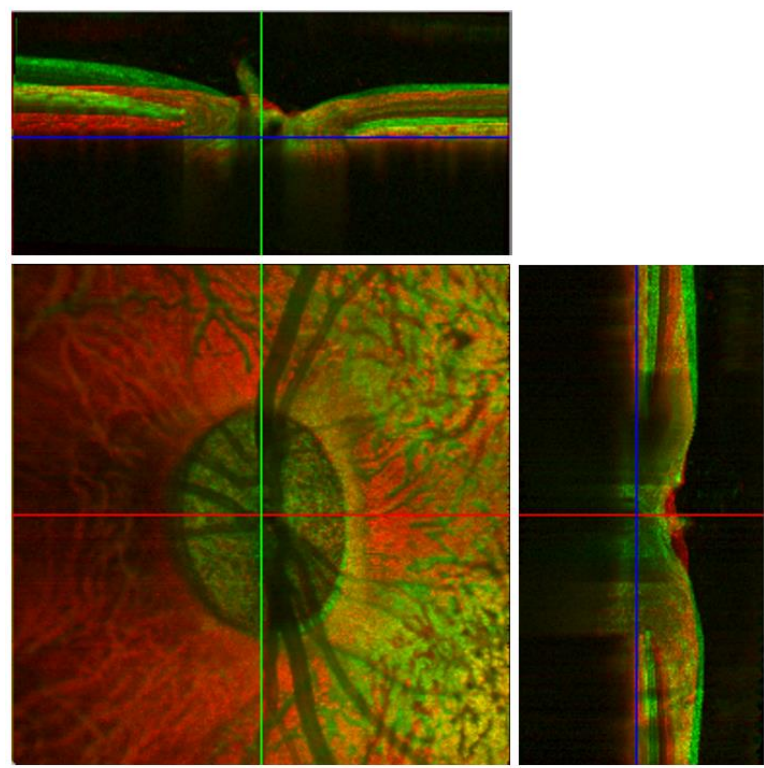

772 Fig. 10. Pre-registration to correct the rigid body motion of the ONH in the OCT volumes acquired

773 before and after elevating the IOP from $10 \mathrm{mmHg}$ to $40 \mathrm{mmHg}$. (a) The mapping before the pre-

774 registration: the red is the reference volume acquired at $10 \mathrm{mmHg}$ and the green is the deformed

775 volume acquired at $40 \mathrm{mmHg}$; (b) The mapping after the pre-registration. Note that these are actual 776 OCT volumes, whereas Fig 7 shows artificial deformations. (b) After pre-registration
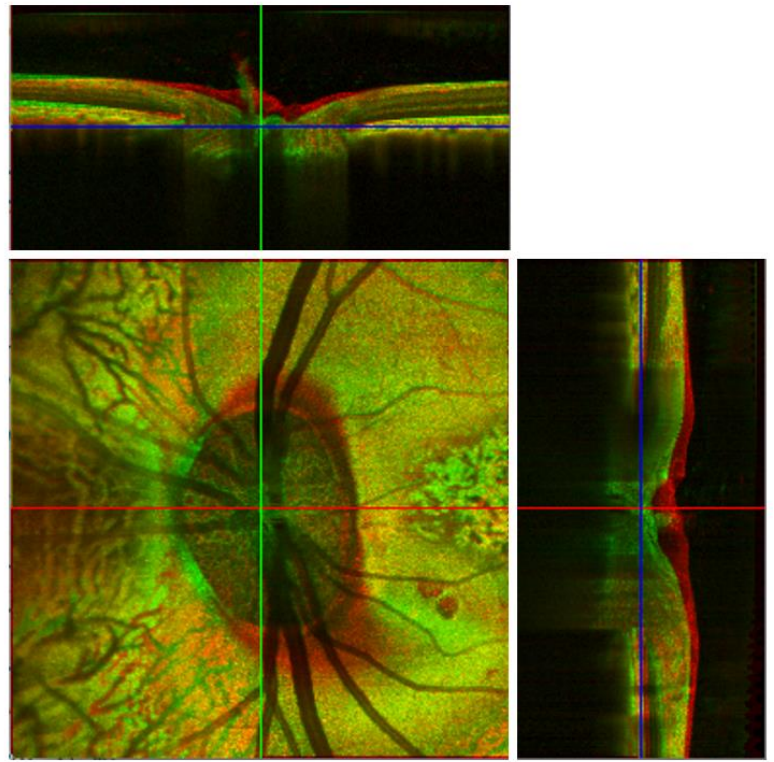

Deformed volume 


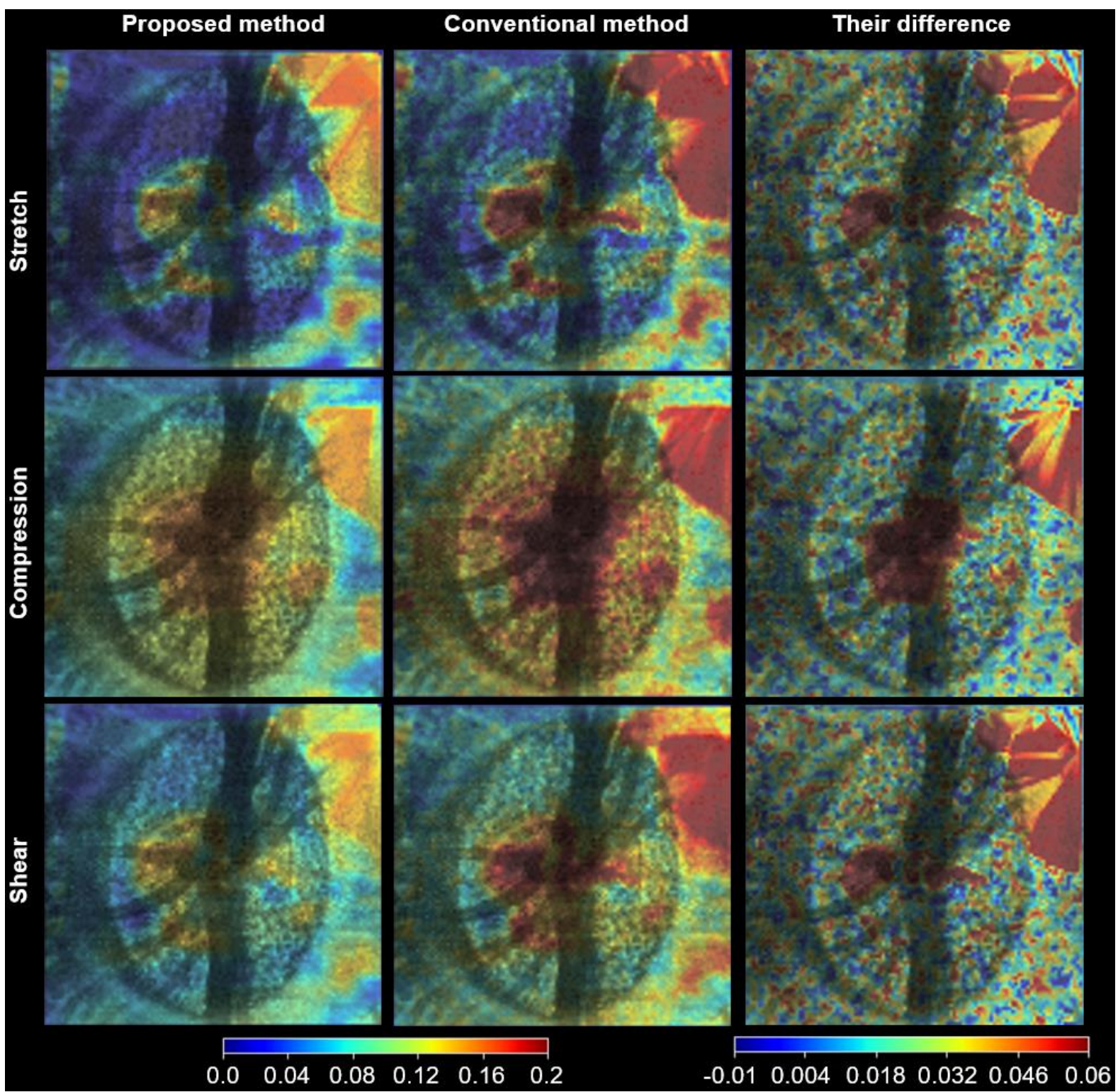

Fig. 11. The full-field stretch, compression, and shear strain of an example C-mode of the OCT volume at the level of the lamina cribrosa induced by elevated IOP from $10 \mathrm{mmHg}$ to $40 \mathrm{mmHg}$. The results in the first column were obtained with the proposed DVC method; the results in the second column were derived with the conventional DVC method; the last column shows their differences: the second column minus the first column (note smaller range spanning both positive and negative). The full-field strains were obtained from the interpolation of the calculated discrete ones. The stretch and compression are the maximal and minimal principal strains, respectively.

Note that compressions are negative strains, but to simplify comparison the results are shown using the absolute values. It is observed that the compression tends to be larger than stretch and shear;

788 on average, the conventional DVC method obtained larger strains than the proposed DVC method.

789 Their strain differences can be up to 0.06 in some regions. The strains from the proposed DVC 
790 method should be more accurate than those from the conventional DVC method considering the

791 fact that 1) strain calculation is very sensitive to noise and the noise often exaggerates the

792 calculated strains; and 2) the proposed DVC method achieved more accurate strains than the

793 conventional method on the test of artificial volumes with preset displacements and strains. From

794 the results of the proposed DVC method, we detected larger strains (up to 0.14) in central lamina

795 and smaller (under 0.04). Notice how the proposed method handled the complex low-signal, high-

796 noise central LC region better than the conventional method for which the local strains become

797 very large, suggesting an error. Both methods can show very large strains in regions where the 798 signal quality was extremely low, for example, outside the canal in the top-right sector. These are

799 an example of a region where it is important to consider both the strength of the correlations and 800 the signal quality before deriving conclusions. 
Table 1. The absolute differences $\left(\Delta T_{x}, \Delta T_{y}, \Delta T_{z}, \Delta \theta_{x}, \Delta \theta_{y}, \Delta \theta_{z}\right)$ between the calculated rigid body motions and the preset ones considering Gaussian and speckle noise

\begin{tabular}{|c|c|c|c|}
\hline \multirow{2}{*}{} & \multicolumn{3}{|c|}{ Rigid-body motion: $\left(T_{x}, T_{y}, T_{z}\right)=(-3.2,1.8,9.3),\left(\theta_{x}, \theta_{y}, \theta_{z}\right)=$} \\
\cline { 2 - 4 } & $\left(5.3^{\circ},-5.8^{\circ},-14.4^{\circ}\right)$ & $\left(-5.3^{\circ}, 5.8^{\circ}, 14.4^{\circ}\right)$ & $\left(-8.3^{\circ}, 7.6^{\circ},-11.2^{\circ}\right)$ \\
\cline { 2 - 4 } & \multicolumn{3}{|c|}{$\left(\Delta T_{x}, \Delta T_{y}, \Delta T_{z}, \Delta \theta_{x}, \Delta \theta_{y}, \Delta \theta_{z}\right)$} \\
\hline $\begin{array}{c}\text { Gaussian } \\
\text { Noise }\end{array}$ & $\begin{array}{c}\left(0.2,0.2,0.3,0.25^{\circ},\right. \\
\left.0.26^{\circ}, 0.36^{\circ}\right)\end{array}$ & $\begin{array}{c}\left(0.2,0.2,0.3,0.16^{\circ},\right. \\
\left.0.14^{\circ}, 0.28^{\circ}\right)\end{array}$ & $\begin{array}{c}\left(0.2,0.2,0.3,0.25^{\circ},\right. \\
\left.0.05^{\circ}, 0.33^{\circ}\right)\end{array}$ \\
\hline Speckle \\
noise & $\begin{array}{c}\left(0.2,0.2,0.3,0.39^{\circ},\right. \\
\left.0.38^{\circ}, 0.19^{\circ}\right)\end{array}$ & $\begin{array}{c}\left(0.2,0.2,0.3,0.26^{\circ},\right. \\
\left.0.18^{\circ}, 0.34^{\circ}\right)\end{array}$ & $\begin{array}{c}\left(0.2,0.2,0.3,0.38^{\circ},\right. \\
\left.0.12^{\circ}, 0.35^{\circ}\right)\end{array}$ \\
\hline
\end{tabular}

804 The unit of translation is voxel.

805

806 